\title{
Stability of the 47 UMa planetary system
}

\author{
K. Goździewski ${ }^{\star}$ \\ Toruń Centre for Astronomy, N. Copernicus University, Gagarin Str. 11, 87100 Toruń, Poland \\ Received 19 February 2002 / Accepted 19 July 2002

\begin{abstract}
In this paper we study the stability of the $47 \mathrm{UMa}$ planetary system in the framework of the $N$-body problem, with application of the MEGNO technique. The method makes it possible to distinguish rapidly between chaotic and regular evolution of a planetary system. Using the initial conditions of the $47 \mathrm{UMa}$ system found by Fischer et al. (2002) we estimate the dynamical bounds on orbital parameters that provide stable motions of the system. We analyze whether a telluric planet can survive orbitally in the habitable zone of the system.
\end{abstract}

Key words. celestial mechanics - stellar dynamics - methods: numerical, $N$-body simulations - planetary systems stars: individual: $47 \mathrm{UMa}$

\section{Introduction}

The search of extrasolar planetary systems conducted extensively over the past 13 years at Lick Observatory led to the recent discovery of the second planet orbiting the star 47 UMa (Fischer et al. 2002). The inner planet b revolves around the parent star with orbital period $P_{\mathrm{b}}=1089 \pm 2.9 \mathrm{~d}$, and the outer companion c has orbital period $P_{\mathrm{c}}=2594 \pm 90 \mathrm{~d}$. This is a unique exosystem, among the more than 100 known up to now, and resembles the Jupiter-Saturn pair in our Solar system. In both cases the planets have almost circular orbits, $e_{\mathrm{b}}=0.061, e_{\mathrm{c}} \in(0,0.2)$, and their orbital periods ratio is close to 5:2. The mass ratios are similar (Fischer et al. 2002), 3.39:1 for 47 UMa and 3.34:1 for Jupiter-Saturn. However, the planets are much bigger and their orbits are much closer than their Solar system counterparts. Thus, their mutual perturbations are stronger than in the Jupiter-Saturn case.

In this work we study the orbital stability of the $47 \mathrm{UMa}$ system and its dependence on the orbital parameters that are unconstrained by the radial velocity observations or not well determined by orbital fits to these data. We also investigate the dynamical structure of the habitable zone of $47 \mathrm{UMa}$ and we try to estimate whether a telluric planet can survive in this zone. This question was already put by Jones et al. (2001). Not knowing about the existence of the second planet, these authors selected the 47 UMa star as one of the best candidates for harboring Earth-like planets.

We explore the orbital parameters' space with the help of the MEGNO indicator (Cincotta \& Simó 2000) ${ }^{1}$. This technique enables rapid determination of whether an investigated

\footnotetext{
* e-mail: k.gozdziewski@astri.uni.torun.pl

1 MEGNO is an acronym for The Mean Exponential Growth factor of Nearby Orbits.
}

initial condition leads to a quasi-periodic or irregular (chaotic) motion of the planetary system. We already applied it when studying the global dynamics of $v$ Andr (Goździewski et al. 2001), HD 82943 (Goździewski \& Maciejewski 2001), and, recently, to the Gliese 876 planetary system (Goździewski et al. 2002). Summarizing the idea briefly, MEGNO $\langle Y\rangle$ is calculated during numerical integration of the equations of motion and the respective variational equations. As was shown by Cincotta \& Simó (2000), if $\langle Y\rangle(t)$ converges to 2 , and after a chosen integration time $t_{\max }$ we have $\left|\langle Y\rangle\left(t_{\max }\right)-2\right|<\epsilon$, (where $\epsilon$ is a small quantity, of the order of $\left.10^{-2}-10^{-3}\right)$, then the tested initial condition leads to a quasi-periodic, stable solution to the system; otherwise it corresponds to chaotic behavior, with a non-zero maximal Lyapunov exponent. MEGNO is related to the maximal Lyapunov exponent $\lambda_{\max }$ through the asymptotic, linear relation

$\langle Y\rangle(t) \simeq a t+b$,

where $a=\lambda_{\max } / 2, b \simeq 0$ for chaotic motion and $a=0$, $b \simeq 2$ for a quasi-periodic solution. This relation makes it possible to estimate efficiently the Lyapunov exponent by the linear fit to $\langle Y\rangle(t)$ (Cincotta \& Simó 2000). The typical motion time ( $t_{\max } \simeq 10^{4}$ orbital periods of the outermost planet), required for the calculation of MEGNO is $10-10^{2}$ times shorter than the time required to estimate the maximal Lyapunov exponent by any classical approach. MEGNO provides computational efficiency, which is crucial in the examination of large sets of initial conditions. Its numerical implementation is also very simple.

Here, MEGNO is utilized for constructing one- and twodimensional sections of the orbital parameters' space in the neighborhood of the initial condition that is determined by dynamical fits to the radial velocity data. Such maps make it possible to find dynamical bounds for those orbital parameters that 

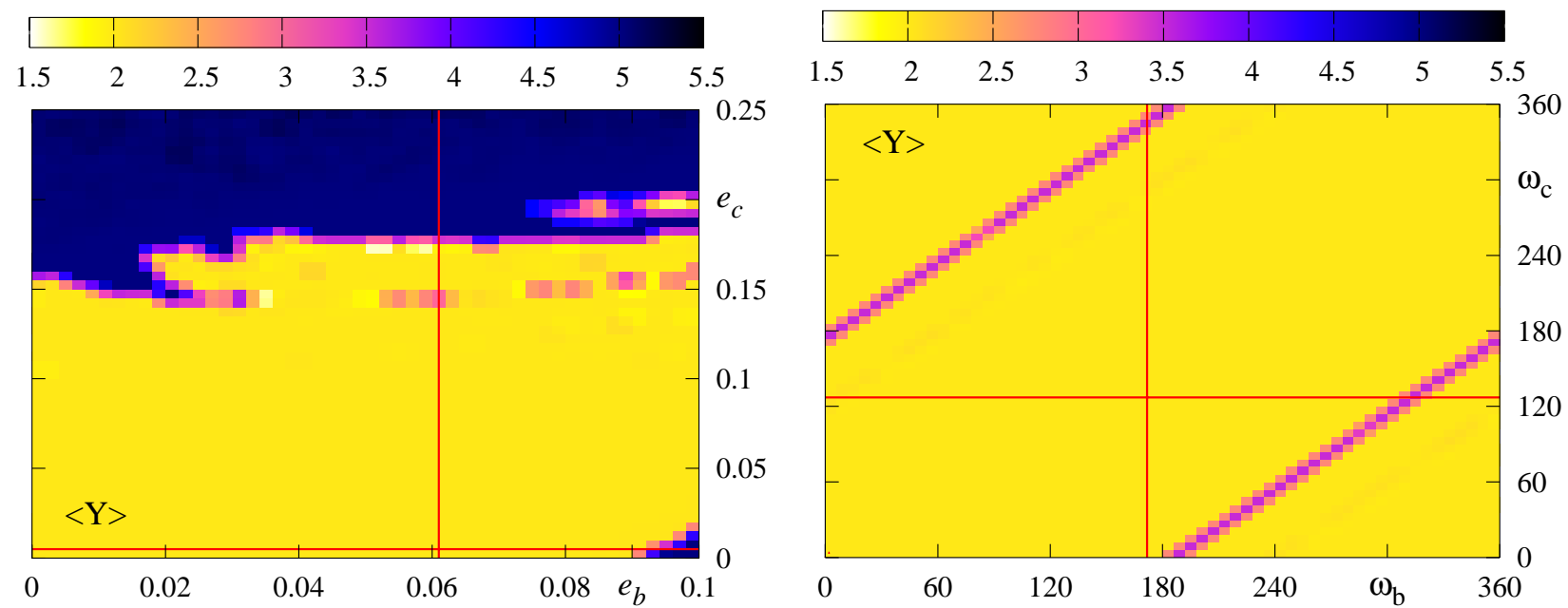

Fig. 1. Stability maps of the 47 UMa system for the initial condition defined in Table 1, and considered as osculating elements. The position of the nominal data is marked with a cross-section of two lines. The data grid in these maps has a resolution of $60 \times 60$ points. Dark shaded areas correspond to chaotic zones and light-gray areas, centered at the level of 2, are for quasi-periodic motions of the system.

are unconstrained by the fits, e.g., the inclination of the system, the masses of the companions ${ }^{2}$, and the relative inclination of orbits. The technique can also be used for efficiently resolving the fine structure of the phase space. For instance, it makes it possible to detect resonances and their widths. Details concerning the application of MEGNO to study the orbital dynamics are given in the papers cited.

A similar methodology of investigating the global dynamics of planetary systems, with the frequency analysis, was applied by Robutel \& Laskar (2001) to the Solar system and to $v$ Andromedae; Michtchenko \& Ferraz-Mello (2001a) used their version of the spectral analysis method to construct dynamical maps of a large portion of the phase space where the outer Solar system evolves. Following these works, and our previous papers (Goździewski et al. 2001; Goździewski \& Maciejewski 2001), we are basically not interested in long-time integrations of the $47 \mathrm{UMa}$ system. Our goal is to find, in the space of initial conditions, regions of chaotic and regular behavior. We also try to determine whether chaotic motions are correlated to changes of orbital elements that appear on a short time scale, of the order of $10^{4}$ orbital periods of the outer companion. Using the same approach we analyze whether the orbit of a telluric planet can survive in the habitable zone of the system.

During the final preparation of this paper, new studies on the 47 UMa planetary system appeared. A paper of Laughlin et al. (2002) concerns the dynamical analysis of the system. The authors investigate its current dynamical state by numerical integration, and compare the results to analytic secular theory. They also study the possibility of Earth-sized objects forming and surviving in the habitable zone of $47 \mathrm{UMa}$.

\footnotetext{
${ }^{2}$ This is not true in general. Incorporating mutual interactions between planets into the model of dynamics that is driving the nonlinear fit procedure, we can remove this ambiguity and the inclinations can also be determined. This procedure was developed by Laughlin \& Chambers (2001), used further by Rivera \& Lissauer (2001), and recently, a new version was presented by Nauenberg (2002).
}

This subject, announced and investigated by Jones et al. (2001), is also studied in recent papers by Thébault et al. (2002), and Noble et al. (2002).

From the technical point of view, in the present paper we give some new examples confirming that MEGNO is an efficient and precise numerical tool for the study of planetary dynamics.

\section{Stability maps of the initial condition}

First we examine the stability of the initial condition of the 47 UMa system found by Fischer et al. (2002). The results of this test are shown on MEGNO stability maps (Figs. 1-3, and 7). The position of the nominal initial condition is marked in the 2D maps with a cross. The initial data seem to lie in a wide stable zone which is seen in the $\left(e_{\mathrm{b}}, e_{\mathrm{c}}\right)-,\left(\omega_{\mathrm{b}}, \omega_{\mathrm{c}}\right)-$, and $\left(M_{\mathrm{b}}, M_{\mathrm{c}}\right)$-planes. The 3D-versions of the stability maps demonstrate that borders between quasi-periodic and chaotic zones are very sharp (Figs. 2 and 3 are typical examples).

MEGNO $\left(a_{\mathrm{b}}, a_{\mathrm{c}}\right)$-map is shown in Fig. 3, and we can read from this map that the nominal initial condition is located closely to the border of unstable zone, which can be identified with the mean motion resonance $7: 3$. Using the initial data from Table 1, we find that the nominal period of planet $\mathrm{b}, P_{\mathrm{b}}=$ 1089 days, corresponds to $a_{\mathrm{b}}=2.0936 \mathrm{AU}$. For a fixed nominal $a_{\mathrm{c}}=3.73 \mathrm{AU}$, the unstable zone begins at $a_{\mathrm{b}} \simeq 2.096 \mathrm{AU}$, and this value is consistent with $P_{\mathrm{b}}=1091 \mathrm{~d}$. MEGNO signature computed for the nominal initial condition (with $a_{\mathrm{b}}=2.09 \mathrm{AU}$ ) is shown in Fig. 9a, and a perfect convergence of $\langle Y\rangle$ to 2 indicates a stable system. For the slightly modified initial condition (with $a_{\mathrm{b}}=2.096667 \mathrm{AU}$ ) we obtain that MEGNO grows linearly, and we estimate the Lyapunov time of this solution at $\simeq 1700 \mathrm{yr}$. In this case, the chaotic behavior can be easily recognized, for example, Fig. 5b shows irregular changes of the inner companion's eccentricity during 1 Myr. Results of a similar calculation that started with the nominal initial data indicate well bounded and regular changes of $e_{\mathrm{b}}$ (see Fig. 5a). 
The fact that the nominal orbits lie very close to the unstable region may be only a coincidence. The errors in the fit are substantial and they can alter the semi-major axes in a wide range: assuming the errors in the periods from Table 1 we obtain that, formally, $a_{\mathrm{b}} \in[2.09,2.1] \mathrm{AU}$, and $a_{\mathrm{c}} \in$ $[3.64,3.82] \mathrm{AU}$. Thus the system can be located in different dynamical zones (see Fig. 3). Moreover, this example contributes to our previous observations (Goździewski \& Maciejewski 2001; Goździewski et al. 2002) indicating that initial conditions, which are found with the fitting process, can be localized closely to unstable zones. Within their error bounds, we can obtain both stable and unstable systems. MEGNO analysis of initial conditions in the vicinity of the fitted parameters helps to estimate efficiently whether the nominal initial conditions are dynamically robust to the fit errors.

Fischer et al. (2002) pointed out that the 47 UMa system resembles the Jupiter-Saturn pair because it resides quite closely to the 5:2 mean motion resonance. The position of this resonance, together with a neighborhood of the nominal initial condition, is shown in Fig. 3. It is known (e.g. Michtchenko $\&$ Ferraz-Mello 2001a) that the 5:2 resonance of Jupiter with Saturn is essentially chaotic. The structure of this resonance was studied in detail by these authors (Michtchenko \& FerrazMello 2001b). The one-dimensional section of the neighborhood of the 5:2 resonance in the 47 UMa system, shown in Fig. 4 , is calculated over $a_{\mathrm{b}}$, with a resolution $10^{-4} \mathrm{AU}$, and for the nominal $a_{\mathrm{b}}=3.73 \mathrm{AU}$. In MEGNO scan one finds a number of extended unstable zones and narrow spikes. These instabilities can be identified with the mean motion resonances. The width of the 5:2 mean motion resonance, as well as the widths of low-order resonances: 7:3 (mentioned already), 9:4, 11:5, can be estimated. Narrow peaks of MEGNO are identified with higher order resonances, labeled in the plot. This figure shows also maximal differences of the values of $a_{\mathrm{c}}$ and $e_{\mathrm{c}}$, reached during the integration time of $\simeq 10^{5} \mathrm{yr}$, with their initial, nominal values. A correlation of these graphs with MEGNO plot, in the semi-major axis ranges related to the low order mean motion resonances 5:2, 7:3, 9:4, and 11:5, is striking. Simultaneously, other peaks of MEGNO that are related to high order resonances do not show such a direct connection with the short-term dynamics of the system.

The stability of the 47 UMa system can be related to the presence of the secular, apsidal resonance in this system (Fischer et al. 2002; Laughlin et al. 2002). This resonance is characterized by librations of the critical angle $\theta_{3}=\varpi_{\mathrm{b}}-\varpi_{\mathrm{c}}$ (where $\varpi_{\mathrm{b}}, \varpi_{\mathrm{c}}$ are the longitudes of periapses), around the line of apsides alignment. Such a mechanism prevents close encounters, and the system can remain in a stable motion (Murray $\&$ Dermott 2000). We show here some examples of the evolution of $\theta_{3}(t)$ in different, potentially possible, dynamical regimes. Figures $6 \mathrm{a}, \mathrm{b}$ illustrate the temporal evolution of eccentricities and the critical angle $\theta_{3}$ at the 5:2 resonance (we selected $a_{\mathrm{b}}=2.004 \mathrm{AU}$ and $a_{\mathrm{c}}=3.73 \mathrm{AU}$ ). The system may also be captured into the 7:3 mean motion resonance, through planetary migration in response to torques from the protoplanetary disk (Laughlin et al. 2002). In this instance the evolution of eccentricities and of the critical angle (see Fig. 6c, d) is qualitatively the same (over a relatively short time) as in the

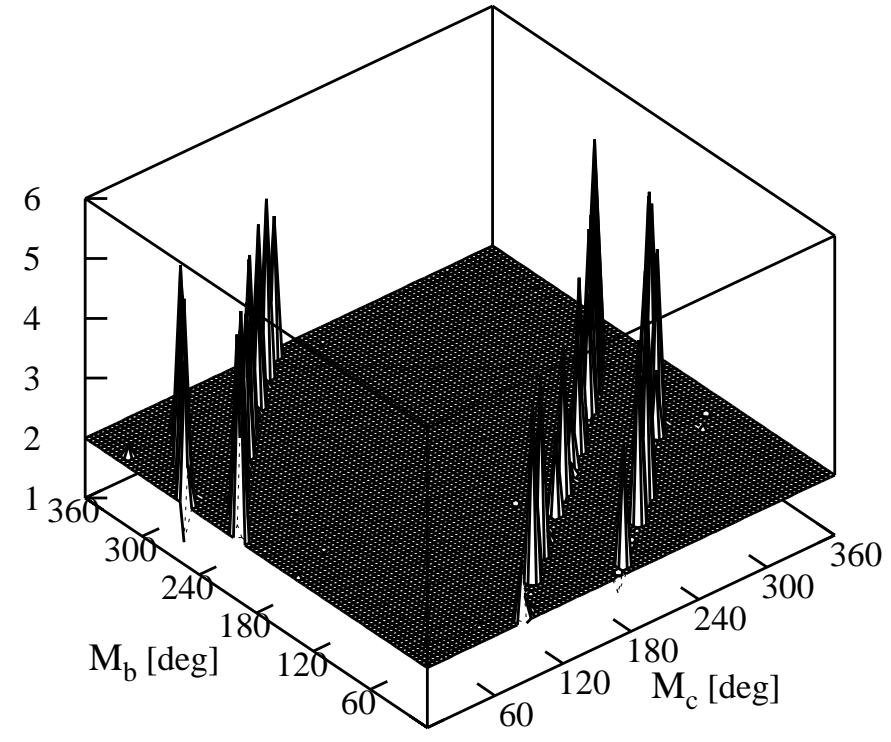

Fig. 2. Stability map of the $47 \mathrm{UMa}$ system on the $\left(M_{\mathrm{b}}, M_{\mathrm{c}}\right)$-plane, computed for osculating elements of epoch A.

nominal system (Fischer et al. 2002, Fig. 2a) (see also Fig. 9a in this paper). The angle $\theta_{3}$ in fact rotates in these two instances, but the apsidal lines remain closely aligned during a relatively long time. This can be a factor stabilizing the motion. (Another, quite peculiar example of the $\theta_{3}$ evolution in a stable configuration of the 47 UMa system is shown in Sect. 3). Note here that the 7:3 resonance appears to be chaotic thus the long-term stability of the system is not likely to be provided in the framework of the $\mathrm{N}$-body model. A more realistic model of the dynamics, incorporating planetary migration and resonance locking, with assumption of the planet-protoplanetary nebula interaction, has been investigated by Laughlin et al. (2002). However, no mechanism is known that can dump the eccentricities efficiently enough to the observed small values, acting against their strong excitation during the resonance locking.

An inspection of the MEGNO map the $\left(\Omega_{\mathrm{b}}-\Omega_{\mathrm{c}}, i\right)$-plane makes it possible to determine the initial, relative inclination of the orbits that provide a stable evolution of the system ${ }^{3}$. For a 2-planet system, the relation between the relative inclination, the inclinations of orbits to the line of sight, and the longitudes of their lines of nodes is given through the following formula:

$\cos \left(i_{\mathrm{rel}}\right)=\cos \left(i_{\mathrm{b}}\right) \cos \left(i_{\mathrm{c}}\right)+\sin \left(i_{\mathrm{b}}\right) \sin \left(i_{\mathrm{c}}\right) \cos \Delta \Omega$,

where $\Delta \Omega=\Omega_{\mathrm{b}}-\Omega_{\mathrm{c}}, i_{\mathrm{b}}, i_{\mathrm{c}}$ are the inclinations of the companions, and $\Omega_{\mathrm{b}}, \Omega_{\mathrm{c}}$ are the longitudes of the lines of nodes. We assume in this test that $i=i_{\mathrm{b}}=i_{\mathrm{c}}$, and MEGNO map is shown in Fig. 7. Note that changes of $i$ alter the companions' masses by the mass factor $f=\sin ^{-1} i$.

${ }^{3}$ If the mutual interaction between planets is not taken into account in the fit model, the relative inclination of planets is not constrained by the radial velocity observations. The reflex-motion of a star that is caused by an isolated planet does not change when an instantaneous orbital plane is rotated along the direction to the observer. A simple superposition of Keplerian orbits does not remove this degeneracy, either. Thus, the position of the orbits' nodes, and their relative inclination, cannot be determined without ambiguity. 



Fig. 3. Stability map of the 47 UMa system for the initial condition defined in Table 1 for osculating epoch A, with the semi-major axes varied. The positions of the mean motion resonances are identified (compare with the one-dimensional scan shown in Fig. 4). A rectangle in the left panel illustrates a formal estimation of the range of semi-major axes, as derived from the errors in the periods. The panel on the right hand side is the $3 \mathrm{D}$-version of the contour plot; it illustrates that borders of chaotic zones are very sharp.

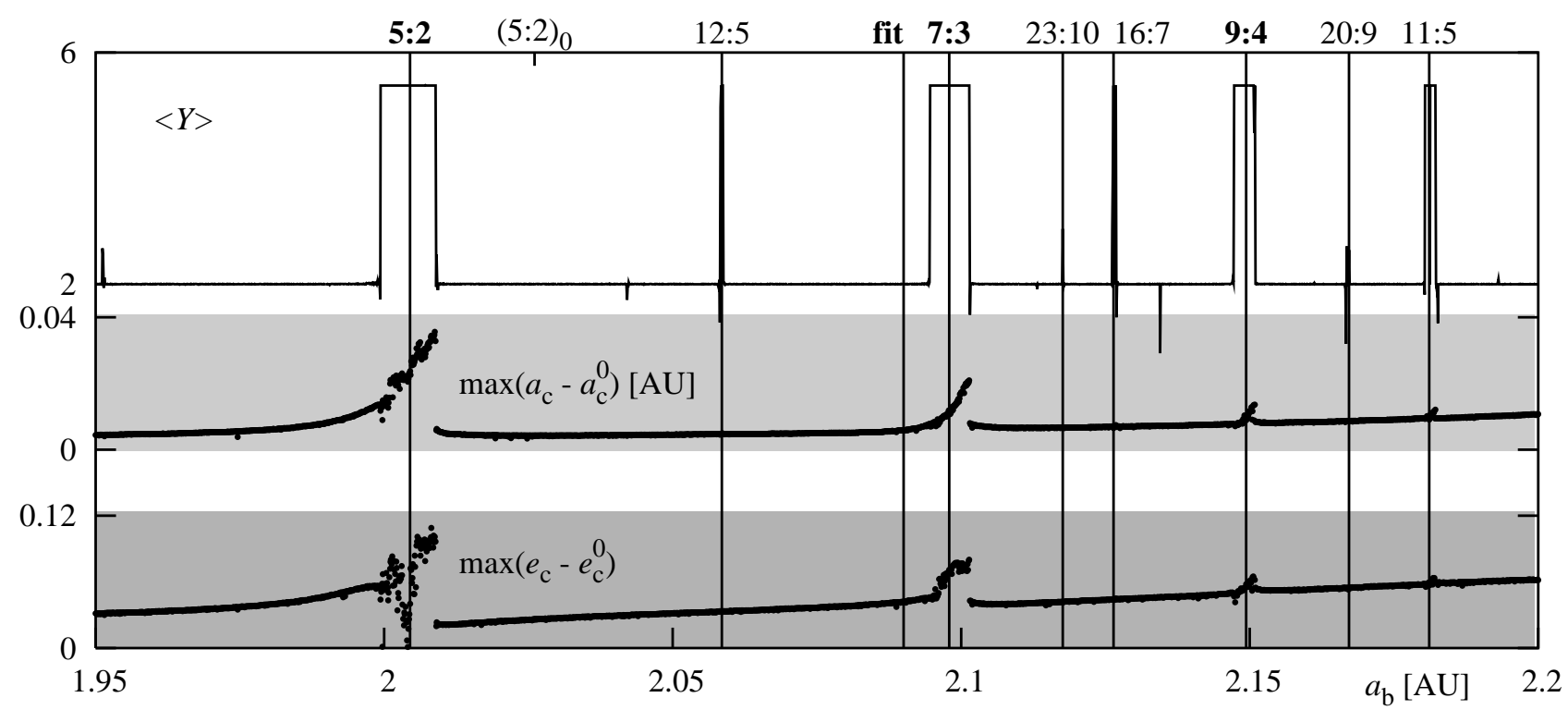

Fig. 4. Scan over $a_{\mathrm{b}}$, with nominal $a_{\mathrm{c}}=3.73 \mathrm{AU}$, of the neighborhood of the nominal initial condition. The upper plot is for MEGNO, the two other plots show the maximal differences of $a_{\mathrm{c}}$, and $e_{\mathrm{c}}$ with their initial values $a_{\mathrm{c}}^{0}, e_{\mathrm{c}}^{0}$, respectively. The resolution of the scan is 0.0001 AU. The labels mark the positions of identified mean motion resonances; label $(5: 2)_{0}$ is for nominal position (i.e., unaffected by the precession of the orbits) of the 5:2 mean motion resonance.

In this map we find some disconnected areas of stable motion. The stability is provided if initially the lines of nodes are aligned (for $i>55^{\circ}$ or $i<40^{\circ}$ ), or anti-aligned (for $i>75^{\circ}$ ). There also exist two narrow zones of stability for $i \simeq 20^{\circ}$ that are permitted for large $\left|\Omega_{\mathrm{b}}-\Omega_{\mathrm{c}}\right|$. These areas are centered roughly at $\pm 120^{\circ}$, and the relative inclinations are $\simeq 40^{\circ}$. Two central zones of stability permit relative inclinations up to $\simeq 60^{\circ}$, and $\simeq 40^{\circ}$, respectively. This result roughly coincides with data derived on the basis of $1 \mathrm{Myr}$ integrations by Laughlin et al. (2002), performed on randomly selected inclinations and angular separation of the nodes. These authors found that the mutual inclination must be smaller than $\simeq 45^{\circ}$, independent of the total mass in the system. Note that in our experiment the planetary inclinations are equal, and this factor can explain the different estimates of the permissible mutual inclinations.

In further tests we assumed that $\Omega_{\mathrm{b}}=\Omega_{\mathrm{c}}=0^{\circ}$.

\section{Dynamical estimates of unconstrained orbital parameters}

A fit to the radial velocity data of $47 \mathrm{UMa}$, performed by Fischer et al. (2002), suffers from significant uncertainties of the time of periastron passage of the outermost planet and its eccentricity. The authors claim that the eccentricity of planet $\mathrm{c}$ is a crucial factor in deciding the system stability. They did numerical three-body integrations extending over $100 \mathrm{Myr}$ and 



Fig. 5. Eccentricity of planet $b$ over $1 \mathrm{Myr}$ for the nominal initial condition (panel a)) and with a small change of the initial semi-major axis $a_{\mathrm{b}}$, from the nominal $a_{\mathrm{b}}^{0}=2.09 \mathrm{AU}$ to $2.096667 \mathrm{AU}$, inside the chaotic zone of the 7:3 mean motion resonance (panel b)). See Fig. 3 for a localization of these data.

a) $5: 2$

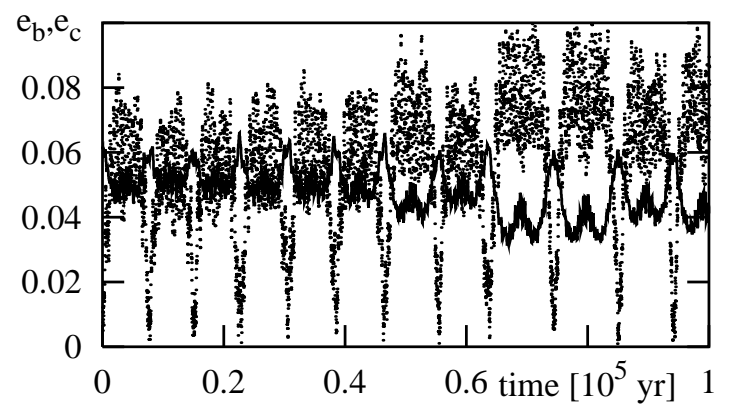

c) $7: 3$

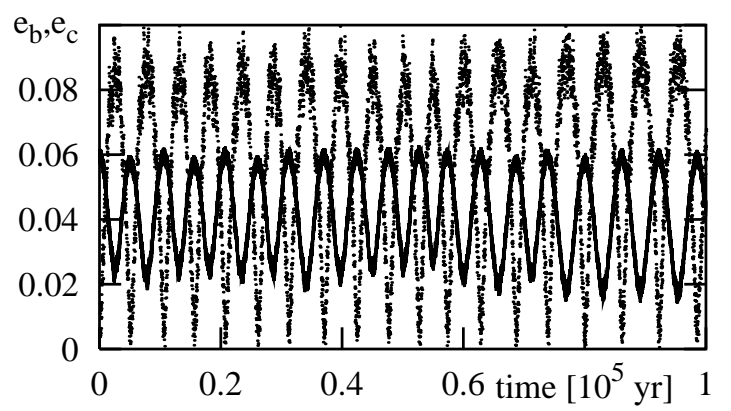

b) $5: 2$



d) $7: 3$

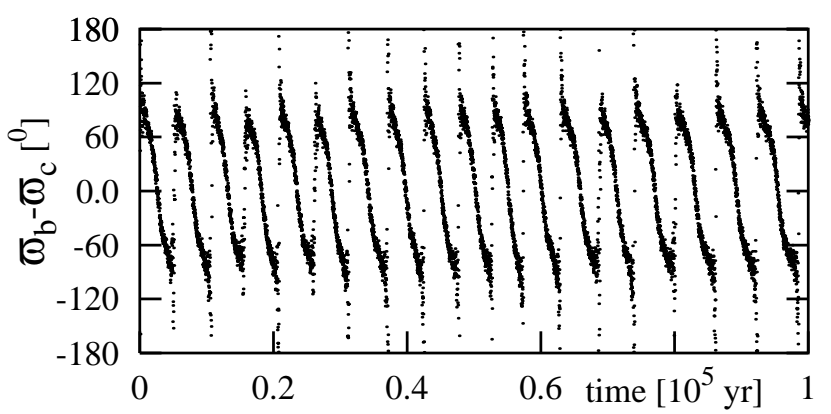

Fig. 6. Evolution of the 47 UMa system at the 5:2 and 7:3 mean motion resonances. Panels a), c) show eccentricities over 0.1 Myr. Panels b), d) illustrate evolution of the critical angle $\theta_{3}$ of the apsidal secular resonance.

concluded that the system is unstable for $\sin i<0.2$ and $e_{\mathrm{c}}>0.2$.

We examined the dependence of the stability on the outer companions' eccentricity with MEGNO. We used two initial epochs for the geometrical elements, and we considered them as osculating elements (see Table 1). Epoch A of JD 2451363.5 is the time of the periastron passage of planet c. Epoch B corresponds to JD 2451293.7 , and was used by the authors as the starting epoch for the long-term integrations that they performed. In a similar test, Laughlin et al. (2002) used epoch A as the initial epoch. For epochs A and B we calculated corresponding companions' mean anomalies, assuming the nominal values of the orbital periods (Table 1). The time of periastron passage of planet $\mathrm{c}$ is determined with a significant error $\simeq 500 \mathrm{~d}$ that corresponds to half the orbital period of companion b. Taking this error into account, we can choose a variety of initial mean anomalies that are consistent with the formal definition of the initial epoch A. In this sense $M_{\mathrm{b}}$ can be varied almost over the whole range $\left[0^{\circ}, 360^{\circ}\right]$, with fixed $M_{\mathrm{c}}=0^{\circ}$. We selected for examination two values of $M_{\mathrm{b}}$, which are related to initial conditions $\mathrm{C}\left(M_{\mathrm{b}}=180^{\circ}\right)$, and D $\left(M_{\mathrm{b}}=60^{\circ}\right)$, respectively.

The results of our integrations are shown in Fig. 8. Plots in the first column, marked with $\langle Y\rangle$, represent the section of the initial parameters' space across the system inclination $i=i_{\mathrm{b}}=$ $i_{\mathrm{c}}$ and the eccentricity of the outer companion $e_{\mathrm{c}}$. Every row in this figure, containing three panels, corresponds to a different initial epoch (and a different combination of the initial mean anomalies).

Panels marked with $\langle Y\rangle$ help us to state that all regions of stable initial conditions are sharply distinguished from areas corresponding to irregular behavior. Although the overall 


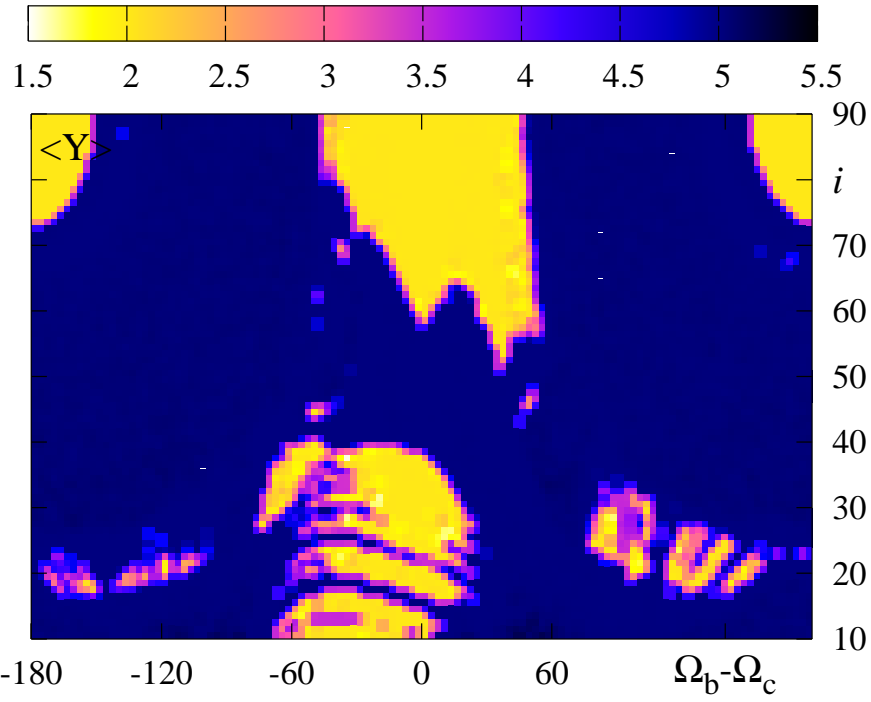

Fig. 7. Stability map of the $47 \mathrm{UMa}$ system for the initial condition defined in Table 1 for epoch A when the system inclination $i$ and initial $\Delta \Omega=\Omega_{\mathrm{b}}-\Omega_{\mathrm{c}}$ are varied.

Table 1. Orbital parameters of the $47 \mathrm{UMa}$ system (Fischer et al. 2002).

\begin{tabular}{|c|c|c|}
\hline Parameter & Planet $b$ & $\overline{\text { Planet c }}$ \\
\hline$m_{2} \sin i\left[\mathrm{M}_{\mathrm{J}}\right]$. & 2.54 & 0.76 \\
\hline$a[\mathrm{AU}] \ldots \ldots$ & 2.09 & 3.73 \\
\hline$\ldots \ldots \ldots \ldots \ldots$ & $1089.0(2.9)$ & $2594(90)$ \\
\hline$e$ & $0.061(0.014)$ & $0.005(0.115)$ \\
\hline$\omega[\mathrm{deg}]$. & $171.8(15.2)$ & $127.0(55.8)$ \\
\hline$T_{\mathrm{p}}[\mathrm{JD}]$ & $2450356.0(33.6)$ & $2451363.5(495.3)$ \\
\hline$M^{\mathrm{A}}(\mathrm{JD} 24513635.3)[\mathrm{deg}]$ & 333.06 & 0.00 \\
\hline$M^{\mathrm{C}}(\mathrm{JD} 24513635.3)[\mathrm{deg}]$ & 180.0 & 0.00 \\
\hline$M^{\mathrm{D}}(\mathrm{JD} 24513635.3)[\mathrm{deg}]$ & 60.0 & 0.00 \\
\hline$M^{\mathrm{B}}(\mathrm{JD} 24512937.7)[\mathrm{deg}]$ & 309.98 & -9.69 \\
\hline
\end{tabular}

distribution of the stability zones is similar for epochs A and B, there exist quantitative differences between them. For instance, the main unstable strip around $i=45^{\circ}$ is significantly narrower for epoch B. For low inclinations (thus, for large masses), the zones of stability are divided by narrow strips of irregular motions. Considering only epoch A and changes of the relative position of the planets, we can observe that for $M_{\mathrm{b}}=180^{\circ}$ (initial condition $\mathrm{C}$ ) the wide, unstable strip is significantly reduced. Finally, for initial condition $\mathrm{D}\left(M_{\mathrm{b}}=60^{\circ}\right)$, the stability zone is almost continuous and uniform. Note that the wide break-up of stability zone seen in Fig. 8a (along line $\Delta \Omega=0^{\circ}$ ), as well as sophisticated patterns of stable and unstable areas for $i<40^{\circ}$, are consistent with the map shown in Fig. 7.

This test shows that the stability of 47 UMa planetary system definitely depends on the value of the initial epoch and on the relative positions of the planets. The reason is that the orbital elements derived from the Doppler observations are not osculating elements, but mean elements. Fischer et al. (2002) checked the importance of neglecting short-term perturbations in the fit to the radial velocity data of $47 \mathrm{UMa}$. They found that discrepancies between the signals derived from Keplerian and full, $N$-body models of the dynamics are at a level of $0.3 \mathrm{~m} \mathrm{~s}^{-1}$ over $14 \mathrm{yr}$ of observations, and are much smaller than the internal precision of the Doppler technique. Moreover, our test with MEGNO suggests that a lack of precise knowledge of the initial epoch has significant dynamical consequences. We cannot, for instance, point out without ambiguity, the proper dynamical bounds of the companions' masses, when we consider the stability in terms of regular and chaotic evolution of the system.

In order to examine the dependence of the system stability on the planetary masses, we computed MEGNO for a few values of the mass factor $f$ and eccentricity $e_{\mathrm{c}}$. The results of this test are shown in Fig. 9. Plots 9a-e are for $\left(i, e_{\mathrm{c}}\right)$-pairs of the initial data, which were examined also by Fischer et al. (2002). Plots in the right column of this figure illustrate variations of the critical angle $\theta_{3}$.

Panel a of Fig. 9 corresponds to a nominal mass factor $f=\sin ^{-1} i=1$ and it gives clear evidence of a quasi-periodic evolution of the system. Panel $\mathrm{b}$ is for $f=1.25$ and the initial condition, localized in the middle strip of a chaotic behavior seen in the $\langle Y\rangle$-panel of Fig. 8 (epoch A). In this instance we have a clear indication of the chaotic system with the Lyapunov time $\simeq 1400 \mathrm{yr}$. The irregular evolution of orbital elements can be easily recognized, for instance in a plot that illustrates $\theta_{3}(t)$. For the same mass factor $f=1.25$ and $e_{\mathrm{c}}=0.1$, we obtain the signature of a regular system, and a clear indication of the presence of the apsidal secular resonance. For $f=3$, which corresponds to $i=19^{\circ} .47$ (see panel e), the system seems to be very close to a regular one - in this case MEGNO converges very slowly (Fig. 9e). (Note that in this case the computations cover $\simeq 1 \mathrm{Myr}$ ). This interpretation is supported by the calculation shown in Fig. 9d; for a slightly changed inclination (and the mass factor $f$ ) we have MEGNO perfectly convergent to the limit of 2 .

In these last two cases the evolution of the critical angle is peculiar. The angle oscillates at levels $\pm 120^{\circ}$ with periodic changes of the libration centers, with a period of about $1000 \mathrm{yr}$. This effect is illustrated in $\theta_{3}$-panels in Fig. 9d, e. The behavior of $\theta_{3}$ is preserved over the integration time $1 \mathrm{Myr}$ and its mechanism is probably a factor that helps to stabilize the system.

The distinction between chaotic and regular motions, which we obtain in a relatively short time scale of MEGNO integrations, appears quite clearly in the time evolution of the orbital elements. In order to illustrate this statement we calculated $\langle Y\rangle$ in the $\left(i, e_{\mathrm{c}}\right)$-plane and, simultaneously, the maximal value of the critical angle of the apsidal secular resonance. These integrations extend over $1.2 \times 10^{4}$ orbital periods of the outer companion: this is long enough to detect the secular resonance. We consider it to be present if $\max \theta_{3}<180^{\circ}$. So we exclude the situation shown in Fig. 5a in (Fischer et al. 2002), as well as in Figs. 6, and 9a: the angle $\theta_{3}$ remains much smaller than $180^{\circ}$ over the integration time, although $\max \theta_{3} \simeq 180^{\circ}$. Analyzing the max $\theta_{3}$-maps, we can confirm that the value of the initial epoch has a significant influence on the global dynamical behavior of the system, and the variations of $\max \theta_{3}$ are correlated with $\langle Y\rangle$. In particular, for initial epochs $\mathrm{A}$ and $\mathrm{B}$, we note a coincidence of the chaotic zone around $45^{\circ}$ with large values of $\theta_{3}$, and "a break-up" of the secular resonance. Let us note that the stability zone is generally more extended than the secular resonance zone, thus the presence of the secular resonance 

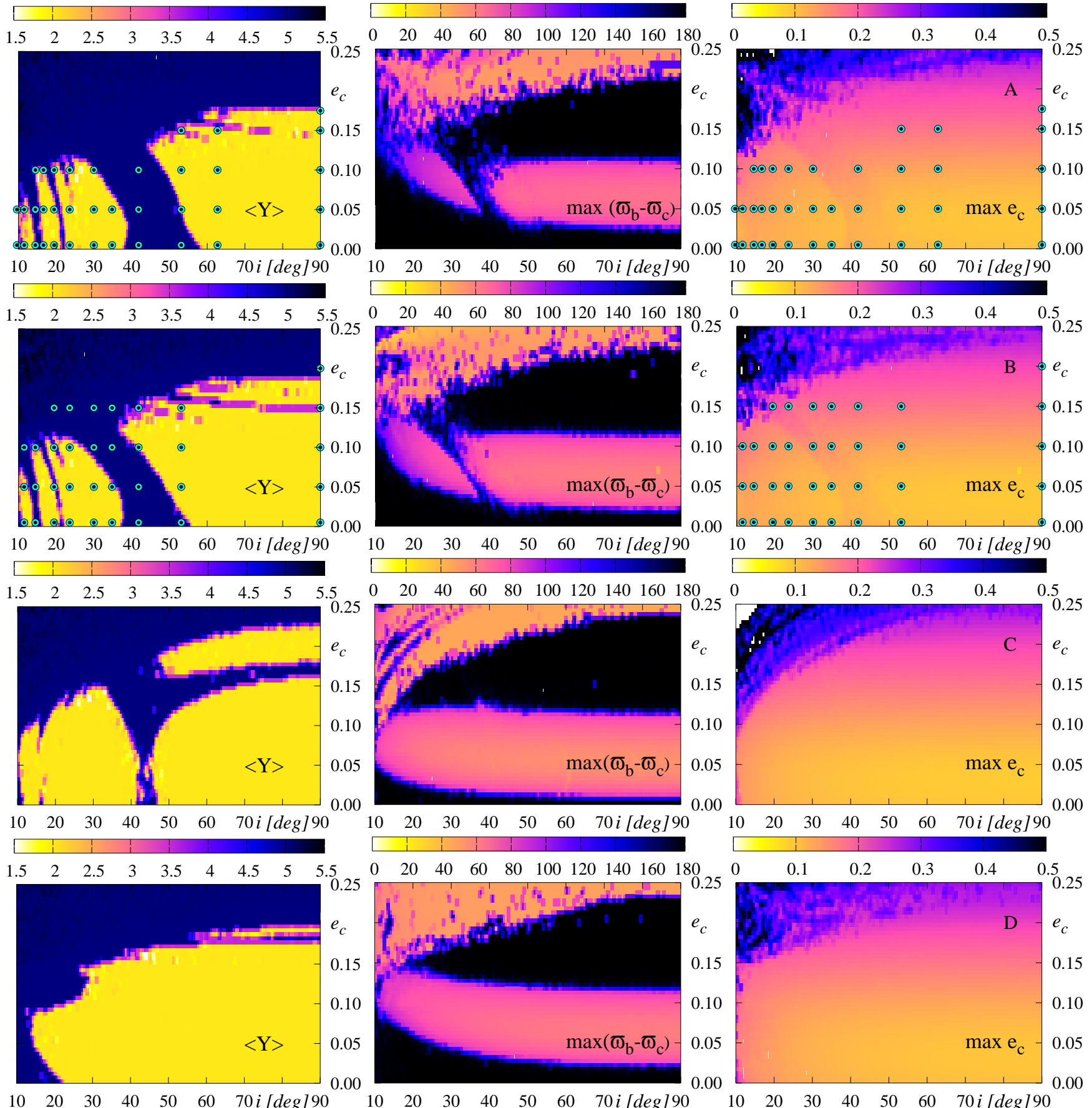

Fig. 8. Influence of the choice of the initial epoch on the geometrical elements of the 47 UMa planetary system. From the top: $M_{\mathrm{b}}=$ $333.06^{\circ}, M_{\mathrm{c}}=0^{\circ}$ (epoch A), $M_{\mathrm{b}}=309.98^{\circ}, M_{\mathrm{c}}=-9.69^{\circ}$ (epoch B), $M_{\mathrm{b}}=180^{\circ}, M_{\mathrm{c}}=0^{\circ}$ (initial condition C which is equivalent to epoch A with an error in the periastron passage of companion c), $M_{\mathrm{b}}=60^{\circ}, M_{\mathrm{c}}=0^{\circ}$ (initial condition D equivalent to other error in the periastron passage of planet c). The plots in the first column illustrate changes of MEGNO when the inclination of the system and the eccentricity of the outer companion are varied. The plots in the middle column correspond to the maximal value of the critical angle of the secular resonance reached in the time span of the integration, $\left(\simeq 10^{5} \mathrm{yr}\right)$. The last column corresponds to the maximum eccentricity of planet c. Small values of $\max \theta_{3}$, for $e_{\mathrm{c}}>0.2$ are due to the very short integration time (of the order of $10^{2} \mathrm{yr}$ ) that is long enough to reach the limit $\langle Y\rangle \simeq 5$, which we set in this test. They are not related to the presence of the secular resonance - in these instances a rapid disruption of the system occurs. The systems reported by Fischer et al. (2002) and Laughlin et al. (2002) as stable, are marked with dotted circles in rows B and A, respectively.

(in the sense adopted for this test) is not necessary to maintain the stability. That supports our previous observation concerning the nominal initial condition.

Analysis of the plots in the last column of Fig. 8 allows us to state that there is a good qualitative agreement of our calculations with the results of the long-term integrations by Fischer et al. (2002) and Laughlin et al. (2002). In these panels we plot the maximal eccentricity $e_{\mathrm{c}}$ that is reached during our integrations. In all of these maps we see that a rough boundary, defined by $e_{\mathrm{c}}>0.2$ and $i>10^{\circ}$ (the mass factor $f<6$ ), 

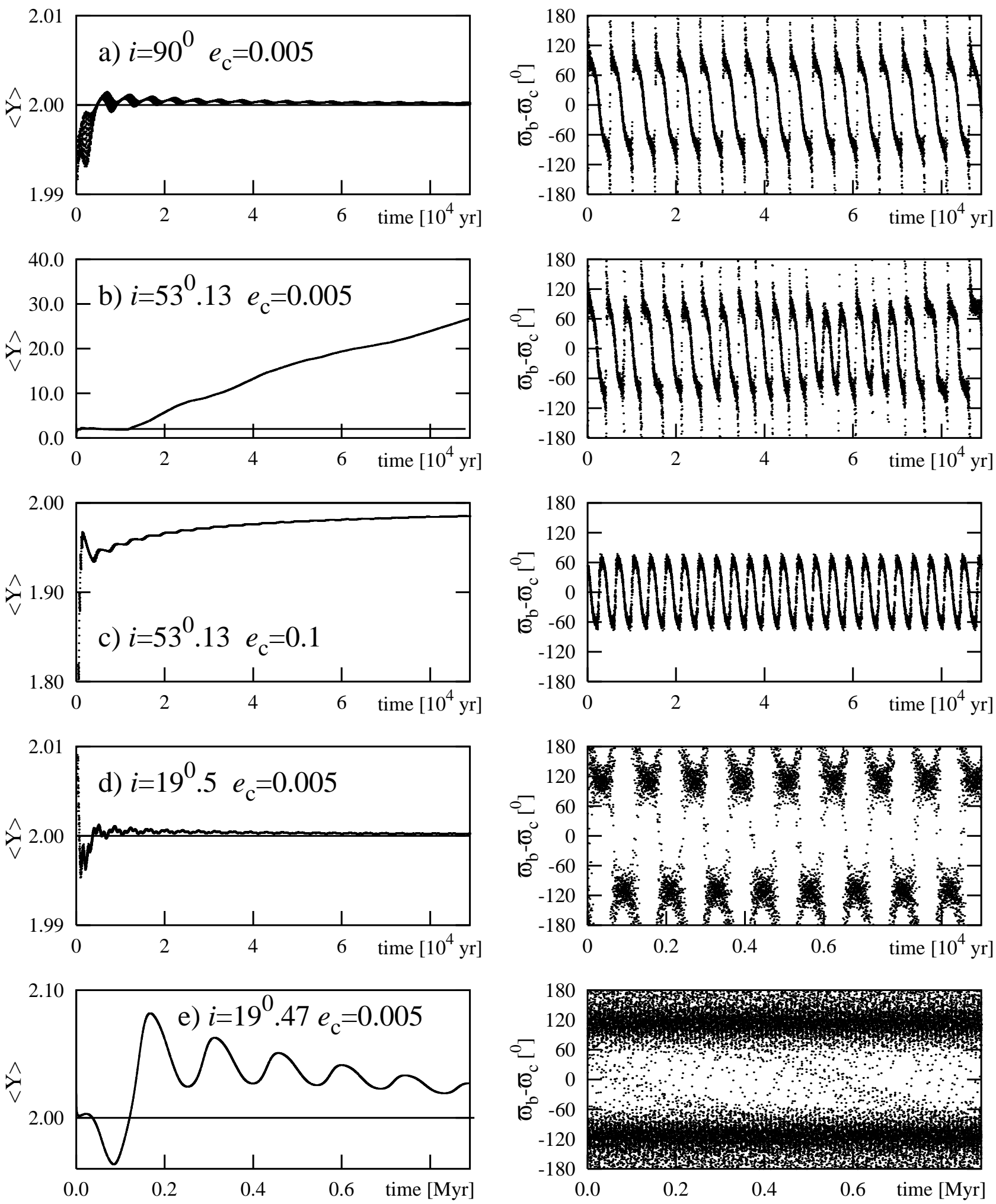

Fig. 9. MEGNO signatures of initial conditions selected from Table 3 of (Fischer et al. 2002). The panels on the left illustrate temporal changes of $\langle Y\rangle$ and the panels on the right show the evolution of the critical angle $\theta_{3}$. Panel a) is for the nominal initial data. Calculations are for osculating epoch A. Note that the time-scale in the last panel, e), is 10 times larger than in previous plots.

coincides with an approximate limit of the long-term stability that is reported by Fischer et al. (2002) (Table 3), and Laughlin et al. (2002) (Table 2). For a reference, positions of systems reported by these authors to survive the 100 Myr integrations are marked in the $\langle Y\rangle$-panels in Fig. 8 by dotted circles.
With a similar test we investigate the dependence of the stability on a combination of the mean anomaly $M_{\mathrm{c}}$ and eccentricity $e_{\mathrm{c}}$ of the outermost planet. (The other orbital parameters are fixed at their nominal values.) The results are shown in Fig. 10, upper row of plots is for epoch A, and lower plots 

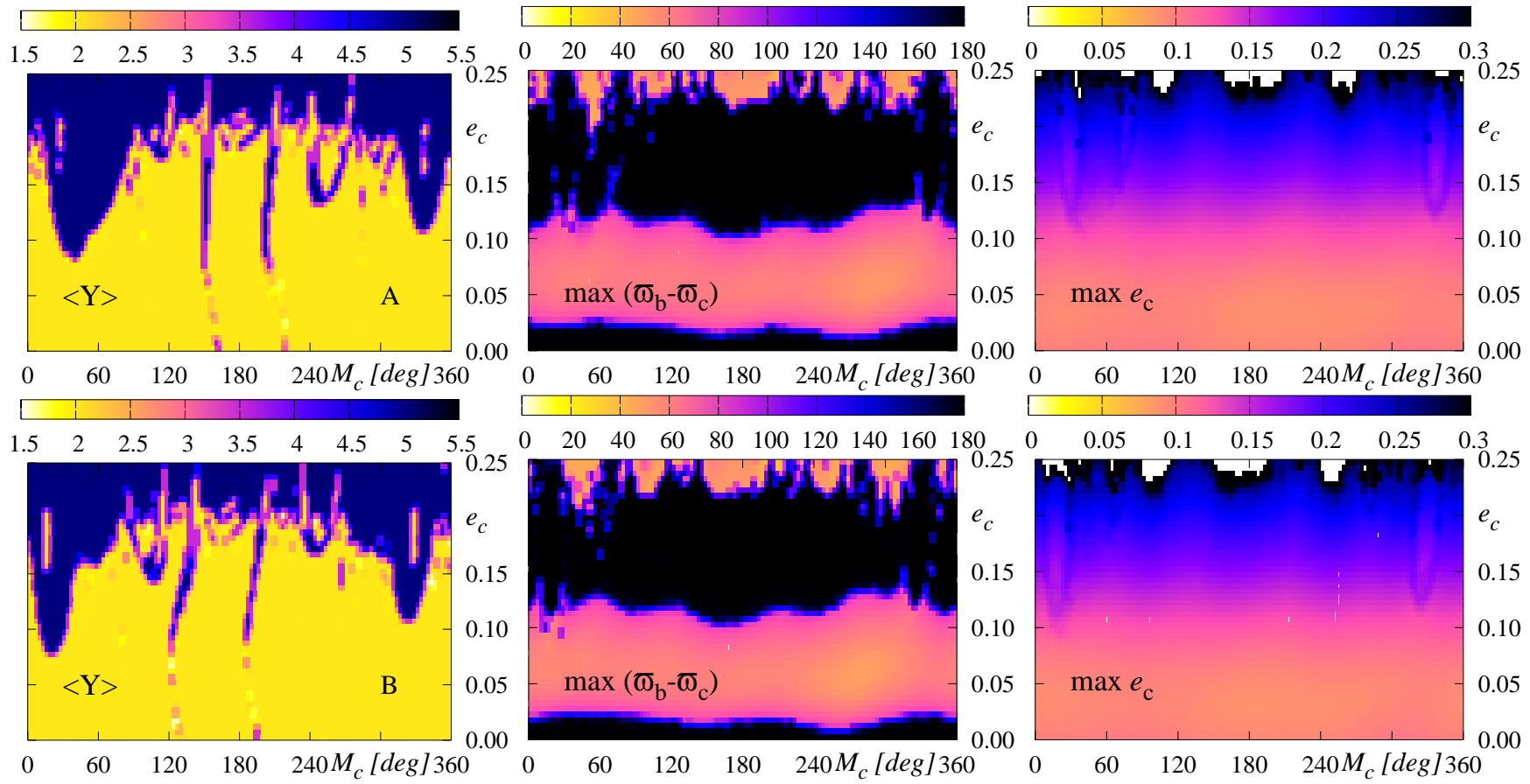

Fig. 10. Stability of the $47 \mathrm{UMa}$ system as function of the mean anomaly and eccentricity of the outer companion. The resolution of the grid is $120 \times 50$ points. The panels in the first column are for $\langle Y\rangle$, those in the middle column for max $\theta_{3}$, and in the third column for max $e_{\mathrm{c}}$.

are for epoch B. Also in this instance we can observe some qualitative differences between corresponding plots. It is common for both epochs that the stability zone shrinks with respect to $e_{\mathrm{c}}$ and for $e_{\mathrm{c}}>0.2$ it disappears completely. Curiously, even for almost circular orbits of planet $c$ there exist very narrow and sharp unstable zones. The secular resonance zones show up clearly in the middle panels of Fig. 10. We can note also a relation between $\langle Y\rangle$ and $\max e_{\mathrm{c}}$.

We finish this section with a short discussion related to the comparison of the long-term integrations of Fischer et al. (2002), Laughlin et al. (2002), and our tests of stability illustrated in Figs. 8 and 9. For all $\left(i, e_{\mathrm{c}}\right)$-pairs, which are marked with dotted circles in Fig. 8b, Fischer et al. (2002) found that the 47 UMa system survives 100 Myr integrations, regardless of whether they are classified with MEGNO as chaotic or regular. A comparison with results of Laughlin et al. (2002) (Fig. 8a) shows a much better coincidence of the stability determinations by the the long-term integrations and MEGNO signatures, although also in this case some systems that survived the integrations are chaotic. (A few such systems are confined to the unstable zone around $i \simeq 45^{\circ}$.) The contradiction can be only apparent, however, because in general there is no obvious correlation between the indication of chaos and a macroscopic instability of motion. Murison et al. (1994) and Holman \& Murray (1996) found that such correlation can exist, indeed, for the Outer Belt asteroids. These authors consider, together with the Lyapunov time $T_{\mathrm{L}}$, the event time $T_{\mathrm{E}}$, i.e., the period of time required for a significant change of orbital elements (an ejection of a body from the system or a collision). The two time scales can differ by many orders of magnitude. An example that is very often quoted concerns asteroid 522 Helga which was initially reported by Milani \& Nobili (1992) as the first case of "stable" chaos. The Lyapunov time of Helga is $T_{\mathrm{L}} \simeq 7000 \mathrm{yr}$ while its $T_{\mathrm{E}}$ is comparable to the age of the Solar system (Holman \& Murray 1996) ${ }^{4}$. Thus the chaotic behavior does not necessarily mean that the orbital evolution of the system is somehow dramatic or that chaos implies a threat to the long-term system stability. Moreover, the $e$-folding time of the Solar system, estimated at the level of 5 Myr (Laskar 1989; Sussman \& Wisdom 1992), is much longer than the Lyapunov times found, in the case of chaotic evolution, for the new exosystems, like $v$ Andr, Gliese 876, or 47 UMa. For these systems $T_{\mathrm{L}}$ is typically of the order of $10^{3} \mathrm{yr}$ only (Laughlin \& Adams 1999; Goździewski et al. 2001, 2002). We cannot exclude that these systems are in fact generically chaotic (Murray $\&$ Holman 2001). One can argue, however, that if the motion of these systems is indeed chaotic, then strong mutual interactions between their members can lead to qualitative changes of the orbits (involving collisions or ejections) in a time-scale much shorter than the analogous time-scale of a few Gyr, found for the Solar system by Laskar $(1994)^{5}$. In this sense the $100 \mathrm{Myr}$ integrations might still be too short to represent the true dynamical evolution of the system when it is chaotic, and to detect possible large instabilities of motion ${ }^{6}$.

\footnotetext{
${ }^{4}$ A dynamical interpretation of short Lyapunov times can be found in Murison et al. (1994). A further explanation of the correlation between $T_{\mathrm{L}}$ and $T_{\mathrm{E}}$ is given in a paper by Shevchenko (1998).

${ }^{5}$ Laskar found that the chaotic diffusion of Mercury is so large that ejection of this planet from the Solar system is possible in less than $3.5 \mathrm{Gyr}$.

6 The results of the stability test performed by Fischer et al. (2002) and in this work can also differ partially because the integration data published by these authors are derived with slightly different initial conditions than finally published (Laughlin et al. 2002), and used in our experiments.
} 
The theory of spacing of planetary systems recently developed by Laskar $(1997,2000)$ can help to investigate such a conjuncture. This theory is based on the notion of the Angular Momentum Deficit (AMD), a quantity that measures the "amplitude of nonlinearity" of motion in an averaged planetary system. In the absence of orbital resonances, the AMD is zero for circular and coplanar orbits, and the system is stable. On the other hand, AMD is large for large values of planetary eccentricities. If the value of AMD is critical, crossing between planetary orbits is possible, and the system becomes unstable. The AMD definition can also be applied to systems that are close to orbital resonances because their orbits are still limited by the conservation of energy and the total angular momentum. As in the secular approximation, large scale chaos allowing planetary escapes corresponds to large values of AMD (Michtchenko \& Ferraz-Mello 2001a). These authors applied the AMD theory to the Outer Solar system and they found that the critical value of AMD (when a disruption of the system occurs in a few million years) corresponds to an eccentricity of Jupiter greater than 0.1, or an eccentricity of Saturn increased to 0.15 . Note that this value is close to the critical eccentricity of planet $\mathrm{c}, e_{\mathrm{c}} \simeq 0.2$, which was found with the long-term integrations by Fischer et al. (2002); Laughlin et al. (2002), and with our MEGNO analysis.

\section{Stability of an Earth-like planet in the habitable zone}

As we have already noticed, $47 \mathrm{UMa}$ is considered as one of the best candidates to host terrestrial planets (Jones et al. 2001). These authors determine the habitable zone of $47 \mathrm{UMa}$ over distances $[0.76,1.43] \mathrm{AU}$ from the parent star, through the requirement of the liquid phase of water (Lissauer 1999). Assuming only one giant companion that was known at the time of writing the paper, the authors showed that the $47 \mathrm{UMa}$ star can harbor Earth-like planets in stable orbits, confined to its habitable zone, over a time scale of the order of $1 \mathrm{Gyr}$. This time-scale is required for forming the biosphere. Their conclusions were based on $1 \mathrm{Gyr}$ numerical integrations of Earth-like Bodies (EB), which are moving in the gravitational field of the giant $b$.

The exploration of the Habitable Zone (HZ) of $47 \mathrm{UMa}$, and a search for possible regions of stability in this area encounters computational difficulties. Direct numerical integrations of motion, even for the simplest three-body system, over a time span of $1 \mathrm{Gyr}$, require huge amounts of CPU time (Jones et al. 2001). Further, it seems difficult to cover with such direct simulations a reasonable range of orbital parameters if we consider a system containing two giants. We do not know the companions' masses and their relative inclinations, and some parameters (like $T_{\mathrm{c}}, e_{\mathrm{c}}$ ) are not well constrained by observations. We have also shown that the 47 UMa system dynamics are sensitive to variation of the planetary masses, and the choice of the initial epoch.

For these reasons, instead of the long-term numerical integrations, we apply again the fast indicator approach. Our aim is to shrink the space of the orbital parameters to that corresponding to quasi-periodic motions of the EB. We cannot claim that the EB certainly would not survive dynamically over $1 \mathrm{Gyr}$ time-scale if its motion was formally classified as irregular. Rather, we bound the possible orbital evolution of the system to regular motions only. These motions do not exhaust all possibilities of bounded orbital dynamics but create a skeleton in the parameters' space certainly filled with stable evolutions. We search also for possible links between the presence of irregular motions, and macroscopic changes of the orbital elements of the EB visible in a short time scale. We select, for tests of the EB stability, configurations of the giants found stable by the previous analysis. Thus we avoid the chaotic evolution of the EB that could be caused by the irregular motion of the primaries.

In the first simulation, we assumed that the giants move in orbits determined by the nominal initial condition defined for epoch A. The system is assumed to be edge-on and coplanar. The EB semi-major axis is varied in the range of $[0.5,1.8] \mathrm{AU}$ with a step $\simeq 0.0007 \mathrm{AU}$. The EB mass is $0.001 M_{\mathrm{J}}$. We set its eccentricity at the initial time to $e_{0}=0$, the longitude of periapse $\varpi_{0}=0^{\circ}$, and the mean anomaly $M_{0}=0^{\circ}$. Variations of MEGNO and orbital elements are calculated over $\simeq 10^{5} \mathrm{yr}$, which corresponds to $1.2 \times 10^{4}$ periods of the outer companion. The results of this test are shown in the upper panel of Fig. 11. The HZ is shaded. MEGNO is marked with thin lines and the lower plot in this figure illustrates maximal values of eccentricity $e_{0}$ reached by the EB during the integration time. (Let us note, that for reason of numerical efficiency, we stop the integrations if MEGNO is larger than 5.)

We find only some very narrow zones of stability in the range of examined semi-major axes corresponding to the $\mathrm{HZ}$. The habitable zone is filled with a large number of MEGNO peaks that correspond to the chaotic motion of the EB. These peaks are related to the mean motion resonances of the EB with the giants, and a number of them can be identified. Labels in the lower row in Fig. 11 describe the mean motion resonances with the inner companion. These resonances are marked with filled circles on the level of $\langle Y\rangle=5$. Similarly, the upper row of labels and open circles corresponds to resonances with the outer planet. (We plotted nominal positions of all resonances of the type $l n_{\mathrm{b}}-k n_{\mathrm{c}} \simeq 0$, where $k=20, l<k$, and $n_{\mathrm{b}}, n_{\mathrm{c}}$ are the mean motions; however, not all of them are labeled.) The $e_{0}$-plot makes it possible to recognize the destabilizing effect of the low-order resonances, e.g., 4:1, 3:1, 2:1, 5:3, 7:4, with the inner giant. In their vicinity, the eccentricity of the EB increases rapidly. In particular, it concerns the neighborhood of $4: 1$ mean motion resonance. During a time less than $10^{5} \mathrm{yr}$, the EB is swept out by the gravitational perturbations of the primaries. In fact, the source of strong chaotic behavior in the area around $0.85 \mathrm{AU}$ (including the zone of the $4: 1$ resonance) is the secular resonance $\varpi_{c}-\varpi_{0}$ that is equivalent to the secular resonance $v_{6}$ in the Solar system, i.e., the commensurability of the precession rate of the asteroid's longitude of perihelion and the average frequency of Saturn's longitude of perihelion (Moons \& Morbidelli 1995). It is known that $v_{6}$ and $v_{5}$ secular resonances $\left(v_{5}-\right.$ with Jupiter) generate strong instabilities of the asteroids motion, in the range of semi-major axes confined to 4:1, 3:1, 5:2, 7:3, and 3:1 mean motion resonances (Moons \& Morbidelli 1995). The presence of $v_{6}$ in the 47 UMa system 

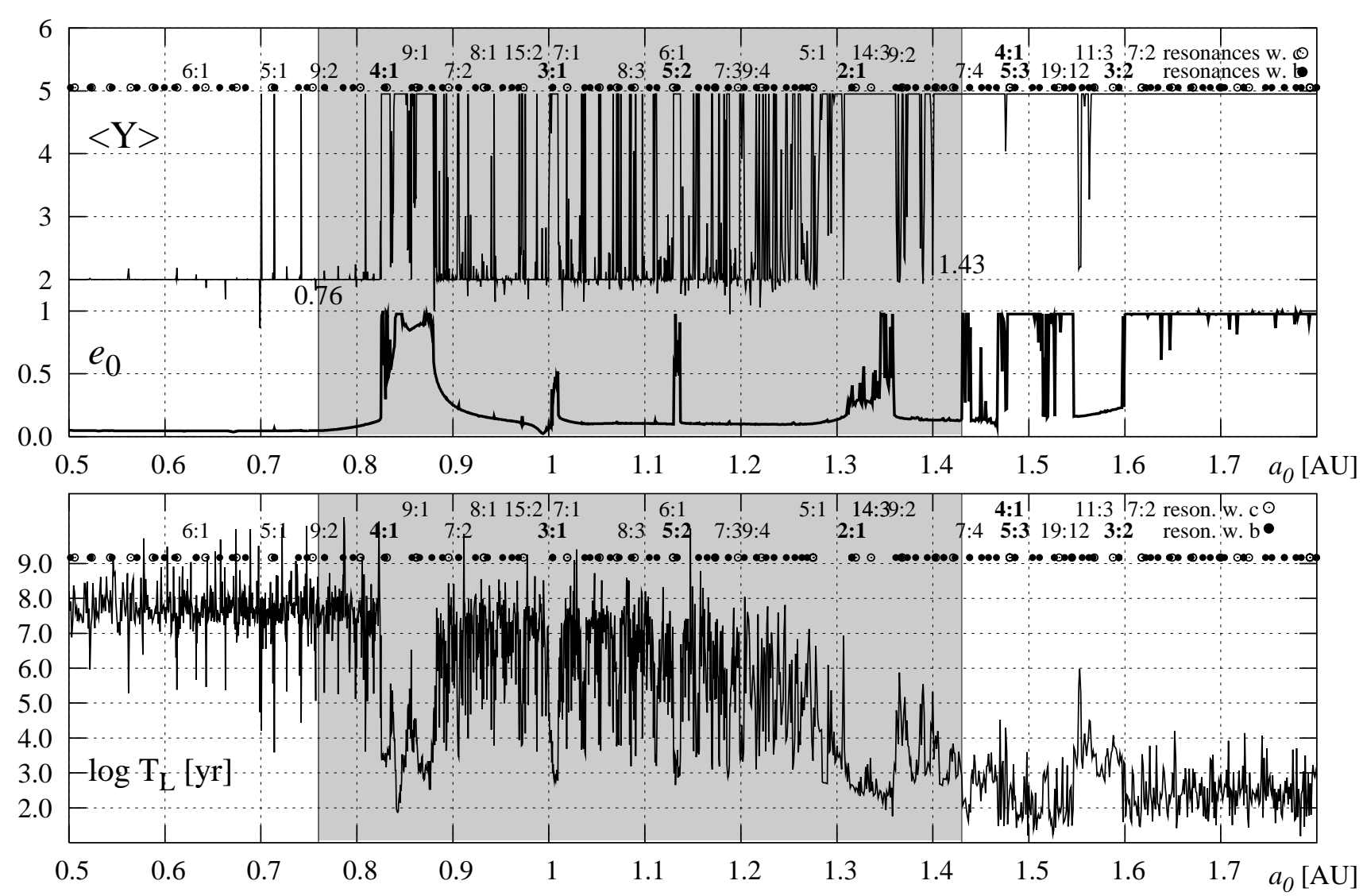

Fig. 11. Stability of an Earth-like body perturbed by the two companions of 47 UMa. Thin lines in upper plot are for MEGNO and thick line illustrates the maximal value of the telluric planet's eccentricity over $10^{5} \mathrm{yr}$. Black circles, labeled in the lower stroke, indicate the mean motion resonances with the inner companion. Open circles and the upper stroke of labels are for the outer planet. The bottom plot shows the estimation of the Lyapunov time $T_{\mathrm{L}}$, derived from the linear fit to $\langle Y\rangle(t)$.

has already been noted by Fischer et al. (2002) and Laughlin et al. (2002).

In the case of $47 \mathrm{UMa}$, a strong instability around $a_{0}=$ 1 AU that corresponds to 3:1 mean motion resonance with the inner planet is correlated to the presence of both the $v_{5}$ and $v_{6}$ resonances (we found both of them). Instability in this region is also noted by Jones et al. (2001). These authors found, however, that the 4:1 mean motion resonance can be stable or unimportant for maintaining the stability, e.g., for $f=1.5$, while our experiments show that this resonance, in the presence of the outer companion, is extremely chaotic. One should remember, however, that the authors were not aware of the outer companion. In order to make the comparison more direct, we computed MEGNO scans for the system containing only planet $b$. Orbital parameters of the test planet and the giant were the same as in the case of the 2-planet system. The results of this simulation are shown in Fig. 12. In this case the vicinity of the $4: 1$ resonance is basically stable, with only one narrow spike of MEGNO, indicating the position of the resonance. The changes of the eccentricity are negligible in the neighborhood of the resonance. It can justify the identification of the source of strong instability, which is present in the 2-planet system in this area, with the resonance $v_{6}$. However, in the regions of $3: 1,5: 2$, and 2:1 resonances the eccentricity still grows rapidly. Basically, the results of this stability test, calculated for a few mass factors, coincide very well with data derived by long-term integrations of Jones et al. (2001).

Considering the 2-planet system, we see that a rapid increase of $e_{0}$ takes place for initial $a_{0}$ confined to the region of 5:2 resonance, and to a wide region of $2: 1$ resonance with the inner planet. The region of stable motions ends definitely at about 1.4 $\mathrm{AU}^{7}$. After this limit, we observe an extended irregular zone. In this zone the eccentricity $e_{0}$ jumps up to 1 for initial $a_{0} \in[1.43,1.55] \mathrm{AU}$, and for $a_{0}>1.6 \mathrm{AU}$, in a very short time of the order of $10^{2} \mathrm{yr}$ only, the eccentricity grows to 1 too; the test planet is removed from the system or collides with the inner giant. A correspondence of isolated peaks of MEGNO with the position of resonances 5:1, 7:2, 8:3, with

\footnotetext{
The resonance overlapping criterion for the circular restricted three body problem predicts large scale chaos for small bodies with initially low-eccentricity orbits for semi-major axes $a_{0} \in\left[a_{\mathrm{P}}-\Delta a, a_{\mathrm{P}}+\right.$ $\Delta a]$, where

$\Delta a \simeq 1.5 a_{\mathrm{P}} \mu^{2 / 7}$

and $a_{\mathrm{P}}$ is the semi-major axis of Jupiter (a giant planet) and $\mu$ is the mass ratio of the giant and the star (Holman \& Murray 1996). Applying this criterion to the inner companion, we found that $\Delta a \simeq$ $0.55 \mathrm{AU}$, thus the chaotic zone predicted by the criterion begins at $\simeq 1.53 \mathrm{AU}$. This value is close to the border of strong instability found with MEGNO.
} 
a) $\mathrm{f}=1$



c) $f=2$

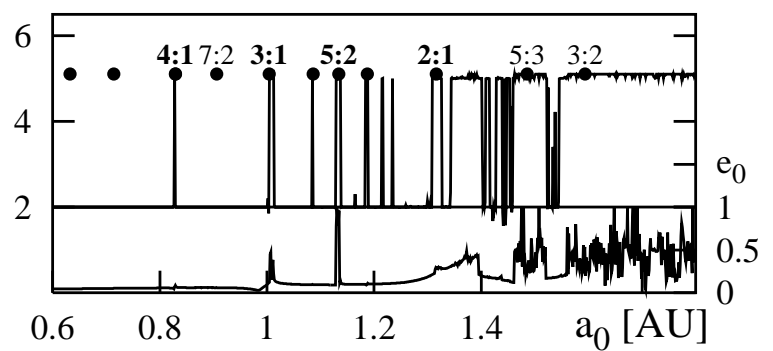

b) $\mathrm{f}=1.5$



d) $f=4$

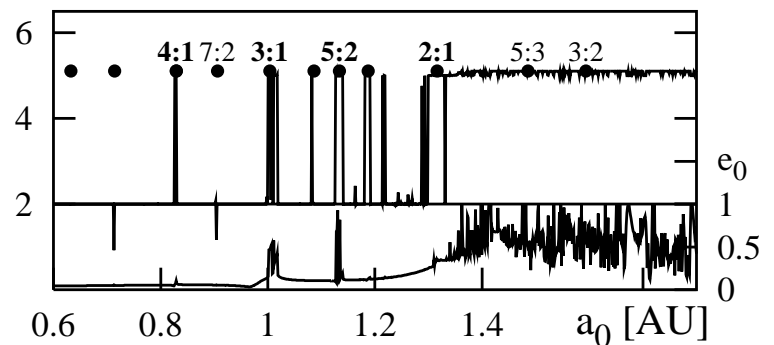

Fig. 12. Stability of an Earth-like body in a single-planet system, perturbed by the inner companion of 47 UMa. Thin lines in the upper plot are for MEGNO and thick line illustrates the maximal value of the telluric planet's eccentricity over $10^{5}$ yr. Black circles, labeled in the lower stroke, indicate the mean motion resonances with the inner companion. Subsequent panels are for mass factors $f=1,1.5,2,4$. The resolution of the scans is 500 data points.

the inner planet, and an overlapping of some resonances, e.g., $5: 3$ with the inner planet and 4:1 with the outer planet, (similarly, 5:2 and 6:1), are also evident. Note also a coincidence of MEGNO spikes with fine changes of $e_{0}$, e.g., in the ranges 0.9-1 AU, and 1.1-1.3 AU. This illustrates that the indicator is very sensitive to changes of the regimes of motion.

The bottom panel of Fig. 11 shows estimations of the Lyapunov times for the system, which are derived through the linear fits to $\langle Y\rangle(t)$, see Eq. (1). For small $a_{0} \in[0.5,0.8] \mathrm{AU}$, we find Lyapunov times on a level of $10^{8} \mathrm{yr}$. In the $\mathrm{HZ}$ the Lyapunov times reach a level of $10^{6}-10^{7} \mathrm{yr}$, but in the chaotic regions they are lowered to as short as $10^{2} \mathrm{yr}$. The zone of strong chaos that begins with the border of the 2:1 resonance, is characterized by very small Lyapunov times, of the order of $10^{2}-10^{3} \mathrm{yr}$ only. This test demonstrates also that estimates of the Lyapunov exponents seem to be more difficult to interpret than indications obtained directly with MEGNO.

We investigated more systematically this simple model of motion of the EB, varying other initial elements of the EB, as well as some parameters of the giants. The results of this experiment are illustrated in Fig. 13. Panels a-d are related to different initial phases of the EB, with respect to the giants; panels $\mathrm{e}-\mathrm{f}$ correspond to orbits of EB inclined to the orbital plane of the primaries by $\simeq 10^{\circ}$. In the tests illustrated in panels $g$, $h$ the system inclination is lowered to $30^{\circ}(f=2)$ and $19.5(f=3)$, respectively. Additionally, we repeated the last two tests for $e_{\mathrm{c}}=0.1$ (panels $\mathrm{i}, \mathrm{j}$ ). The range of semi-major axes examined in this experiment is somewhat narrower than in the first test (see Fig. 11), but it overlaps almost all the HZ. As in the previous test, the initial eccentricity of the EB is always $e_{0}=0$ and its orbit is slightly inclined by $0.1^{\circ}$ to the orbital plane of the giants' system, which is considered to be co-planar. Together with MEGNO, we examined the maximal eccentricity attained by the orbit of the EB during the period of integration. Note that it is relatively short, at most $\simeq 1.2 \times 10^{4} P_{\mathrm{c}}$, because the time span of the calculations is determined by the limit $\simeq 5$, given to MEGNO. In every run illustrated in the panels of Fig. 13, the initial configuration of the primaries corresponds to regular evolution, and is based on the nominal data from Table 1.

Although the initial conditions of the EB are varied substantially in these experiments, the motion of the EB exhibits some common properties. The HZ is densely filled with peaks of MEGNO that can be identified (similarly to the previously analyzed case), with a number of low-order mean motion resonances. Some of them, which have a strong influence on the motion of the EB, have already been identified. Resonances $4: 1,3: 1,5: 2,2: 1$, with the inner giant, and the $v_{5}, v_{6}$ secular resonances have a particularly disrupting character. The eccentricity of the EB is forced to values of the order of 0.1 , even in a few narrow zones of quasi-periodic motions. This effect of forced eccentricity of the EB was also reported by Jones et al. (2001). Large masses of the primaries, see panels $\mathrm{g}-\mathrm{j}$, cause the unstable zones to be much more extended than for the case of minimal masses of the giants; only a few narrow zones or regular motions are preserved. We observe also that a rough center of the strong instability caused by the $v_{6}$ resonance is shifted to 0.9AU for inclined configurations of the EB (Fig. 13e, f), and to $0.95-1 \mathrm{AU}$ for massive configurations of the giants (Fig.13, panels $g-j$ ).

Further, we analyzed the effect of the not well known eccentricity of companion $c$ on the motion of the EB. This test 
is calculated on the $\left(a_{0}, e_{\mathrm{c}}\right)$-plane for the nominal initial condition describing motions of the giants. We repeated it for $m_{0}=10^{-10} M_{\mathrm{J}}, m_{0}=10^{-3} M_{\mathrm{J}}$, and $m_{0}=5 \times 10^{-3} M_{\mathrm{J}}$. The results are shown in Fig. 14. In all these cases we obtain qualitatively the same distribution of stable and unstable zones. This indicates that, basically, the overall distribution of stable zones is insensitive to moderately low masses of the EB. We repeated the test for $m_{0}=10^{-3} M_{\mathrm{J}}$ with a better resolution (Fig. 15), and simultaneously, we evaluated the maximal $e_{0}$ of EB during the integrations. The correlation of chaotic zones with regions of high maximal eccentricities is striking. Again, these zones can be identified with the neighborhoods of the mean motion resonances with the inner planet (e.g., 4:1, 3:1, 5:2, 2:1), and the secular resonances $v_{5}, v_{6}$. One-dimensional cross-sections of the $\left(a_{0}, e_{c}\right)$-plane, for a few fixed values of eccentricity $e_{\mathrm{c}}$, with resolution 500 points, illustrate this statement very clearly, see Fig. 16. Stable motions end definitely at $e_{\mathrm{c}} \simeq 0.18$, and this value corresponds to the global instability of the system already investigated.

Finally, we did a scan of MEGNO, by fixing the initial condition of the giants as the nominal one (with osculating epoch A), and varying the semi-major axis and the eccentricity of the EB simultaneously. The results are shown in Fig. 17. In this test the strong instabilities caused by the mean motion and the secular resonances, which we observed in the previous experiments, are also evident. The initial eccentricities of the EB that permit regular motion depend strongly on initial values of $a_{0}$, and for $a_{0}>1.3 \mathrm{AU}$ the regular zones almost completely disappear. We also observe a clear correlation of large maximal eccentricities of the EB achieved during the integration time with the distribution of regular and chaotic zones.

These experiments suggest that the dynamical environment in the habitable zone of $47 \mathrm{UMa}$ for surviving a telluric planet is not favorable. The structure of the phase space in the HZ recalls the analogy of the Asteroid belt in the Solar system. The mean motion resonances with Jupiter cleared some parts of this region from small bodies, leaving prominent gaps in the distribution of their orbital elements. Variations of orbital elements of Jupiter caused by Saturn can further destabilize trajectories in resonance regions (Lissauer 1999; Murray et al. 1998). A similar dynamical mechanism seems to be supported in the $47 \mathrm{UMa}$ system by their two main members. Orbital migration of the giants can be another factor shifting unstable resonances and causing the strongest instabilities in the motion of small telluric bodies.

Moreover, the current orbital state of the giants permits some narrow regions of the semi-major axes (e.g., around 0.93$0.96 \mathrm{AU}$, or $1.05 \mathrm{AU}$ ), which correspond to quasi-periodic motions of the EB. Let us also note a very extended zone of stable motions existing for $a_{0} \in[0.3,0.6]$ AU. Fischer et al. (2002) claim that most test particles survive in the $\mathrm{HZ}$ over 1 Myr time-scales. Possibly, outside the resonance regions, the motion of the test particles is bounded even if it is chaotic. This conclusion is supported by numerical simulations of Laughlin et al. (2002). These authors found that test particles survive the $20 \mathrm{Myr}$ integrations outside the resonance regions.

In the dynamical environment of the $\mathrm{HZ}$ of $47 \mathrm{UMa}$, a formation of a large telluric planet by accretion of material left after forming a migrating giant (Jones et al. 2001; Murray et al. 2002) does not seem likely either. Assuming the scenario of the accretion theory, Kortenkamp \& Wetherill (2000) analyzed the mechanism of forming inner Solar system planets. They found that when both gas drag and Jovian perturbations are important, different-sized planetesimals follow slightly eccentric orbits which are out of phase with one another. Collisions between the objects are energetic enough to prevent accretion. The magnitude of this effect is sensitive to the orbits of the giant planets. If Jupiter formed quickly, the mechanisms hinder both the growth time scales for the terrestrial planets and formation of large members of the asteroid belt. This mechanism is likely to act in the habitable zone of the $47 \mathrm{UMa}$ system. Additionally, the sweeping effects of the unstable mean motion and secular resonances can lead to quick removal of a large portion of the protoplanetary material.

These conclusions, and the results of the dynamical analysis of the motion of the test bodies, are in very good agreement with the results of independent work by Laughlin et al. (2002) and Thébault et al. (2002). The accretion simulations of Laughlin et al. (2002) show that it is unlikely that Earth mass planets could have formed in the HZ of 47 UMa unless very massive planetary embryos accreted prior to the formation of the giant planets. Thébault et al. (2002), considering the inner giant only, have found that this planet excites large eccentricities among the planetesimals in the $\mathrm{HZ}$ preventing accumulation. There is only a chance for accretion in a small region around the star inside 0.3 AU. These authors conclude that the outer companion does not significantly affect the encounter velocity distribution in the inner regions of the system. They found that beyond $0.8 \mathrm{AU}$ planetesimals or planetary embryos are rapidly removed by perturbations of the giants.

Although a detailed search for telluric planets in the $\mathrm{HZ}$ of $47 \mathrm{UMa}$ is a fascinating subject, it is beyond the scope of this paper. We think that more light on the dynamical environment in this zone can be provided by an application of the existing theory which explains the dynamics of the asteroid belt (Duncan \& Quinn 1993; Holman \& Murray 1996; Murray et al. 1998; Lissauer 1999, and references therein).

\section{Conclusions}

In this work we performed a dynamical analysis of the initial conditions of the 47 UMa system found by Fischer et al. (2002) by 2-planet Keplerian fit to the Doppler velocities of the star. If the stability of the system is understood in terms of chaotic and quasi-periodic evolution, these initial conditions cannot be used as osculating elements. We tried to estimate the ranges of the orbital elements (unconstrained by the radial velocity observations) which provide stable, quasi-periodic evolution of the system. A change of initial relative phases of the planets in the range of a few tens of degrees permitted by the range of the fit errors, leads to significant ambiguities in these estimations. The fit errors of the time of periapse passage of the outermost planet and its eccentricity remove constraints on the dynamical state of the system. The analysis of the orbital stability performed by Fischer et al. (2002), and Laughlin et al. (2002), with the application of the long-term numerical integrations, is compared 
a) $e_{\mathrm{c}}=0.005 \quad i_{0}=89.9^{0} \quad \omega_{0}=0^{0} M_{0}=0^{0} \lambda=0^{0}$

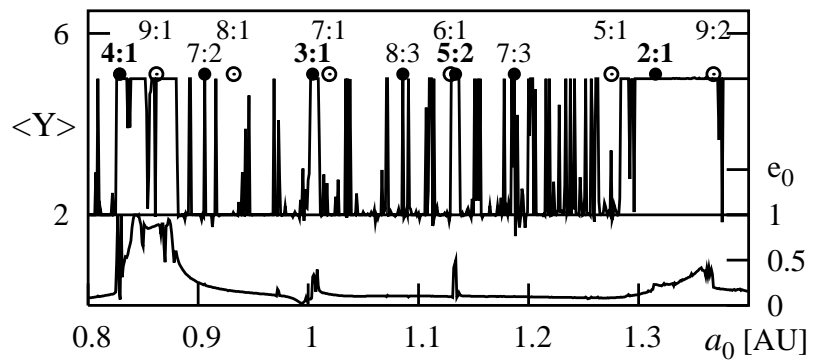

c) $e_{\mathrm{c}}=0.005 \quad i_{0}=89.9^{0} \quad \omega_{0}=0^{0} M_{0}=120^{0} \lambda=120^{0}$

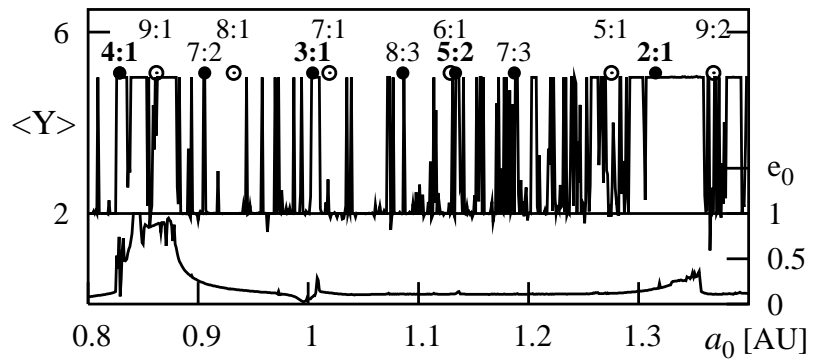

e) $e_{\mathrm{c}}=0.005 \quad i_{0}=79.9^{0} \quad \omega_{0}=90^{0} M_{0}=0^{0} \lambda=90^{0}$



g) $e_{\mathrm{c}}=0.005 \quad i=30^{0} \quad \omega_{0}=90^{0} M_{0}=90^{0} \lambda=180^{\circ} \mathrm{f}=2$

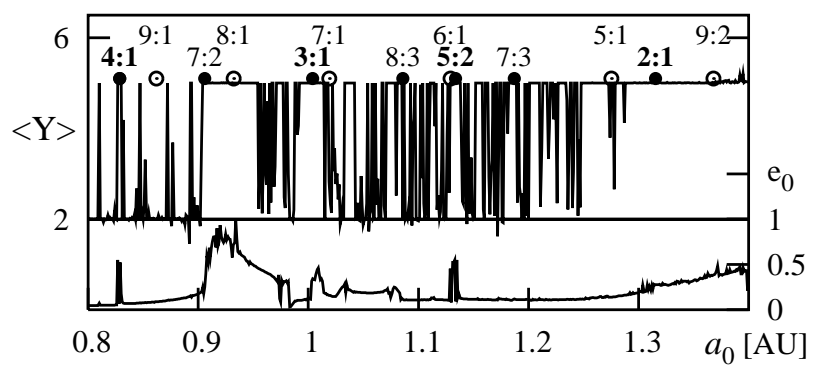

i) $e_{\mathrm{c}}=0.100 \quad i=30^{0} \quad \omega_{0}=90^{0} M_{0}=90^{0} \lambda=180^{\circ} \mathrm{f}=2$

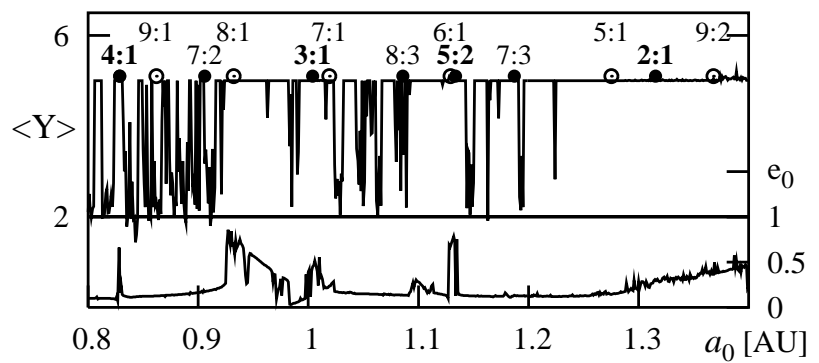

b) $e_{\mathrm{c}}=0.005 \quad i_{0}=89.9^{0} \quad \omega_{0}=90^{0} M_{0}=0^{0} \lambda=90^{0}$

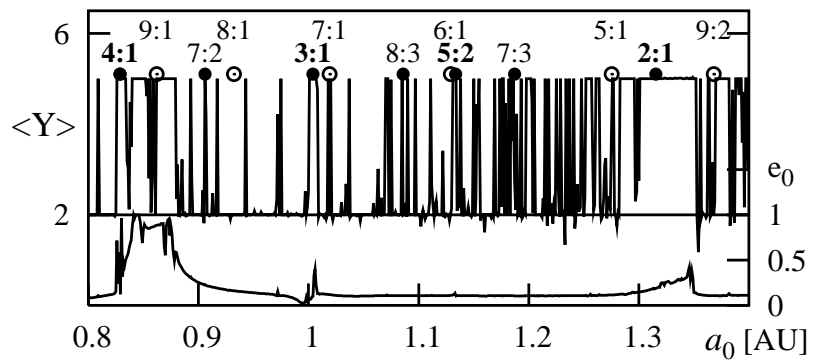

d) $e_{\mathrm{c}}=0.005 \quad i_{0}=89.9^{0} \quad \omega_{0}=90^{0} M_{0}=120^{0} \lambda=210^{0}$

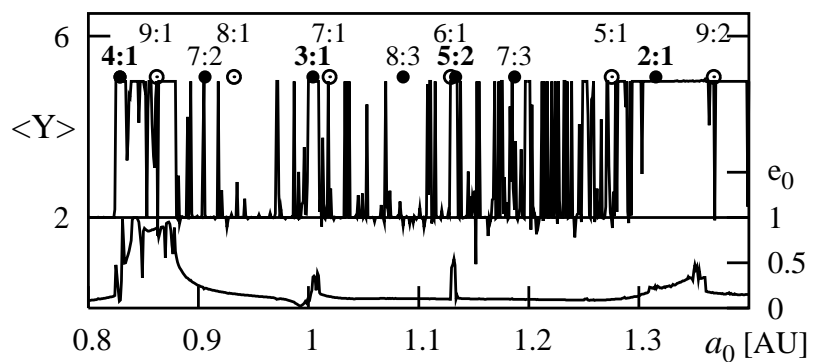

f) $e_{\mathrm{c}}=0.005 \quad i_{0}=79.9^{0} \quad \omega_{0}=90^{0} M_{0}=120^{0} \lambda=210^{0}$



h) $e_{\mathrm{c}}=0.005 \quad i=19.5^{0} \quad \omega_{0}=90^{0} M_{0}=0^{0} \lambda=90^{0} \mathrm{f}=3$

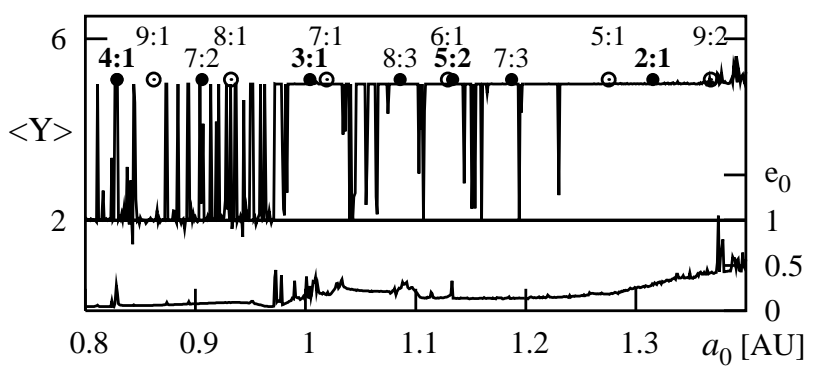

j) $e_{\mathrm{c}}=0.100 \quad i=19.5^{0} \quad \omega_{0}=90^{0} M_{0}=0^{0} \lambda=90^{0} \mathrm{f}=3$

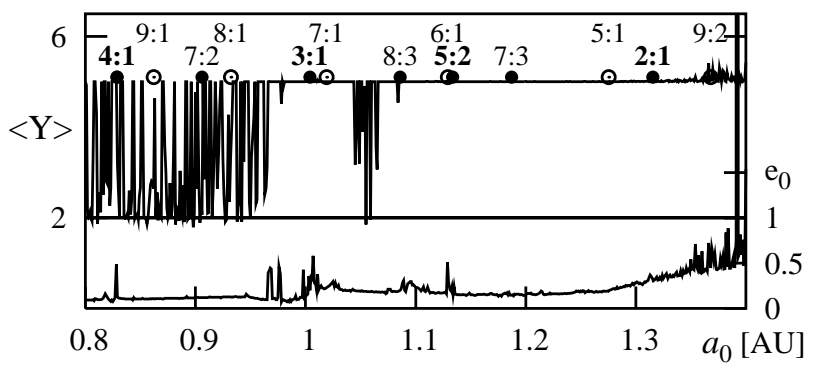

Fig. 13. One-dimensional scans of $\langle Y\rangle$ and the maximal eccentricity $e_{0}$ of the Earth-like body, moving in the habitable zone of $47 \mathrm{UMa}$. In every panel the upper graph is for MEGNO and the lower graph is for eccentricity. Panels are labeled with initial conditions of the Earth-like body chosen for a given test. $\left(i_{0}\right.$ is the inclination of the EB, $\varpi_{0}$ is the longitude of periapse, $M_{0}$ is the mean anomaly, and $\lambda$ is the longitude). Initial conditions of the giant planets are given in Table 1 . The resolution of the plots is $0.001 \mathrm{AU}$. See the text for explanation. 


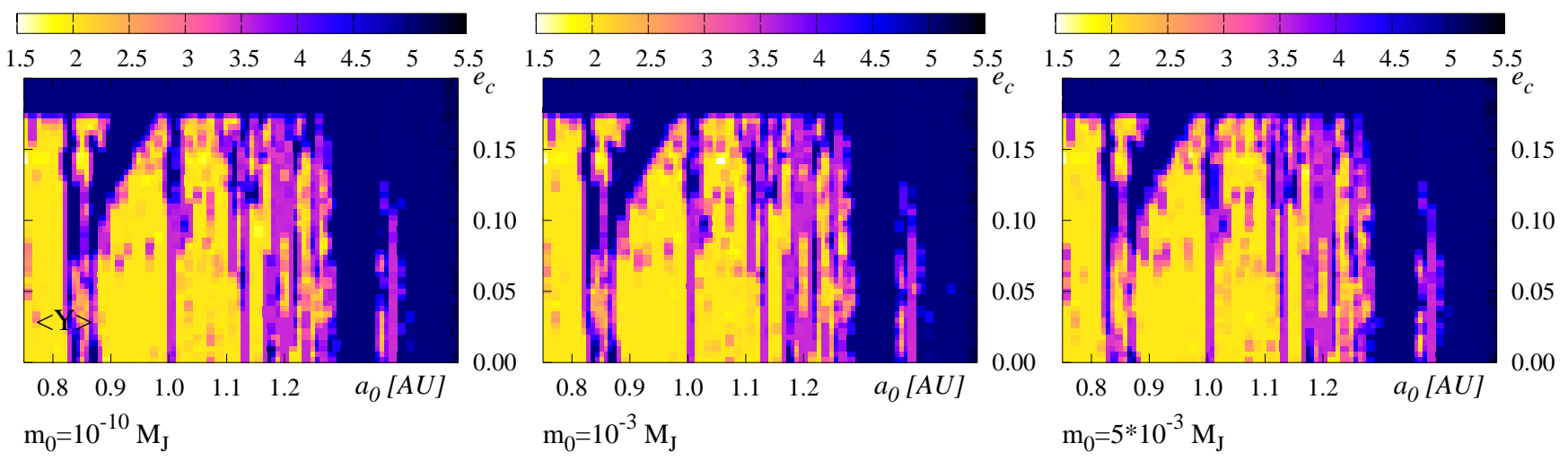

Fig. 14. Stability maps in the plane of $\left(a_{0}, e_{c}\right)$ for different masses of a telluric planet. The left panel is for $m_{0}=10^{-10} M_{\mathrm{J}}$, the middle panel is for $m_{0}=10^{-3} M_{\mathrm{J}}$, and the right panel is for $m_{0}=5 \times 10^{-3} M_{\mathrm{J}}$. The resolution in the $x$-axis is 100 points and in the $y$-axis it is 50 points.


Fig. 15. Stability map for a telluric planet with mass $m_{0}=10^{-3} M_{\mathrm{J}}$. The initial parameters of its orbit are $e_{0}=0, \omega_{0}=0^{\circ}, M_{0}=90^{\circ}$. The initial condition for the primaries is the nominal one. The data resolution is $150 \times 50$ points.

with MEGNO analysis. We found that some systems, which survived the $100 \mathrm{Myr}$ period of integration, have a chaotic character, with short Lyapunov time-scale, $\simeq 10^{3} \mathrm{yr}$ only. This does not necessarily contradict the long-term bounded motion of the 47 UMa planets, but the significance of this chaos for the longterm dynamics is not understood completely and needs further explanation. In some cases the occurrence of chaotic motions is tightly correlated with dynamical effects appearing in the short time scale $\simeq 10^{5} \mathrm{yr}$ of MEGNO integrations.

Analyzing the neighborhood of the nominal initial condition found by Fischer et al. (2002), we found that it can be located in the close vicinity of unstable zones. This observation supports our previous conclusions (Goździewski \& Maciejewski 2001; Goździewski et al. 2002) that the dynamical analysis of the fits to the radial velocity data can help to estimate whether the fits are dynamically robust.

Using the initial conditions of stable systems found with MEGNO, we analyzed the dynamical structure of the habitable zone of $47 \mathrm{UMa}$. It is densely filled with unstable zones related to the mean motion and secular resonances with the giant members of the system. Due to the dynamical mechanisms created and supported by the giants in this region, formation of a large telluric planet is not likely. There exist many dynamical analogies of the habitable zone of 47 UMa with the Asteroid belt in the Solar system. We think that the problem of the existence and survival of an Earth-like body in this zone can be further investigated in the framework of the dynamical theory of the Asteroid belt.

Our work gives some new examples that confirm the usefulness of the MEGNO indicator for studies of planetary dynamics.

Acknowledgements. I am very grateful to Dr. Andrzej J. Maciejewski for discussions and comments that improved the manuscript. I am very grateful to the referee, Dr. John Chambers, whose remarks have been vital for improving the manuscript. Many thanks to Zbroja for correcting the manuscript. I would like to thank the Editor, Jet Katgert for extensive language revision of the final version of the manuscript. The computations that were required for this work were done partially on a high performance PC computer donated by The Alexander von Humboldt Foundation to the Torun Centre for Astronomy, and on Sun Enterprise E6000 system hosted by Regional Multiprocessor Laboratory of Nicolaus Copernicus University. This work is supported by the KBN Research Grant No. 2P03D 00122. 
a) $\mathrm{e}_{\mathrm{c}}=0.005$

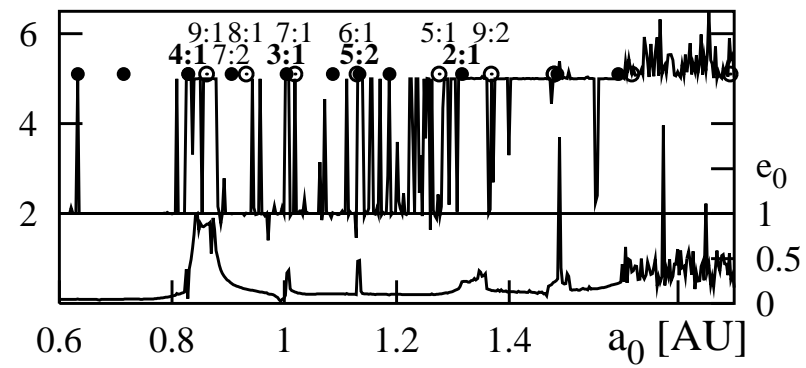

c) $\mathrm{e}_{\mathrm{c}}=0.15$



b) $\mathrm{e}_{\mathrm{c}}=0.1$

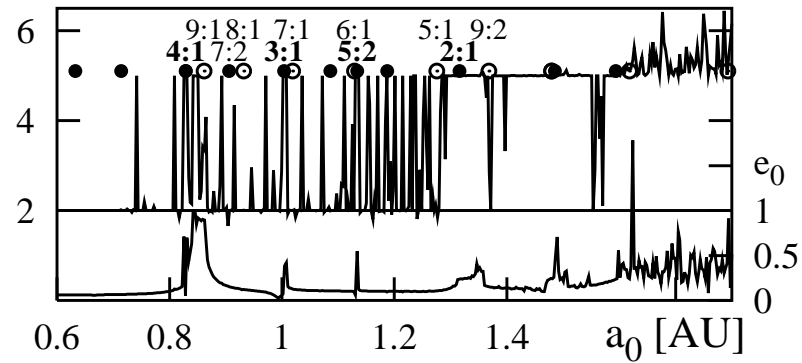

d) $e_{c}=0.2$



Fig. 16. One-dimensional cross-sections of the $\left(a_{0}, e_{\mathrm{c}}\right)$-plane for fixed eccentricities of planet $\mathrm{c}$. The meaning of the open and filled circles, as well as the labels is the same as in Fig. 11.


Fig. 17. Stability map of the Earth-like body in the plane of its initial semi-major axis $a_{0}$ and eccentricity $e_{0}$. The plot on the left is for $\langle Y\rangle$, and the plot on the right illustrates the maximal value of the eccentricity reached during the integration time.

\section{References}

Cincotta, P. M., \& Simó, C. 2000, A\&AS, 147, 205

Duncan, M. J., \& Quinn, T. 1993, ARA\&A, 31, 265

Fischer, D. A., Marcy, G. W., Butler, R. P., Laughlin, G., \& Vogt, S. S. 2002, ApJ, 564, 1028

Goździewski, K., Bois, E., \& Maciejewski, A. 2002, MNRAS, 332, 839

Goździewski, K., Bois, E., Maciejewski, A., \& Kiseleva-Eggleton, L. 2001, A\&A, 378, 569

Goździewski, K., \& Maciejewski, A. 2001, ApJ, 554, L81
Holman, M. J., \& Murray, N. W. 1996, AJ, 112, 1278

Jones, B. W., Sleep, P. N., \& Chambers, J. E. 2001, A\&A, 366, 254

Kortenkamp, S. J., \& Wetherill, G. W. 2000, Icarus, 143, 60

Laskar, J. 1989, Nature, 338, 237

Laskar, J. 1994, A\&A, 287, L9

Laskar, J. 1997, A\&A, 317, L75

Laskar, J. 2000, Phys. Rev. Lett., 84, 3240

Laughlin, G., \& Adams, F. C. 1999, ApJ, 526, 881

Laughlin, G., Chambers, J., \& Fischer, D. 2002, ApJ, in press

Laughlin, G., \& Chambers, J. E. 2001, ApJ, 551, L109

Lissauer, J. J. 1999, Rev. Mod. Phys., 71, 835 
Michtchenko, T., \& Ferraz-Mello, S. 2001a, ApJ, 122, 474

Michtchenko, T. A., \& Ferraz-Mello, S. 2001b, Icarus, 149, 357

Milani, A., \& Nobili, A. M. 1992, Nature, 357, 569

Moons, M., \& Morbidelli, A. 1995, Icarus, 114, 33

Murison, M. A., Lecar, M., \& Franklin, F. A. 1994, AJ, 108, 2323

Murray, C. D., \& Dermott, S. F. 2000, Solar System Dynamics (Cambridge Univ. Press)

Murray, N., \& Holman, M. 2001, Nature, 410, 773

Murray, N., Holman, M., \& Potter, M. 1998, AJ, 116, 2583
Murray, N., Paskowitz, M., \& Holman, M. 2002, ApJ, 565, 608 Nauenberg, M. 2002, ApJ, 568, 369

Noble, M., Musielak, Z. E., \& Cuntz, M. 2002, ApJ, 572, 1024

Rivera, E. J., \& Lissauer, J. J. 2001, ApJ, 402, 558

Robutel, P., \& Laskar, J. 2001, Icarus, 152, 4

Shevchenko, I. I. 1998, Phys. Lett. A, 241, 53

Sussman, G. J., \& Wisdom, J. 1992, Science, 257, 56

Thébault, P., Marzari, F., \& Scholl, H. 2002, A\&A, 384, 594 